\title{
Unfold/fold transformations preserving termination properties
}

\author{
Torben Amtoft \\ Computer Science Department \\ Aarhus University \\ Ny Munkegade, building 540 \\ DK-8000 Århus C, Denmark \\ nternet: tamtoft@daimi.aau.dk
}

August 19, 1992

\begin{abstract}
The unfold/fold framework constitutes the spine of many program transformation strategies. However, by unrestricted use of folding the target program may terminate less often than the source program. Several authors have investigated the problem of setting up conditions of syntactic nature, i.e. not based on some well-founded ordering of the arguments, which guarantee preservation of termination properties. These conditions are typically formulated in a way which makes it hard to grasp the basic intuition why they work, and in a way which makes it hard to give elegant proofs of correctness. The aim of this paper will be to give a more unified treatment by setting up a model which enables us to reason about termination preservation in a cleaner and more algebraic fashion. The model resembles a logic language and is parametrized with respect to evaluation order, but it should not be too difficult to transfer the ideas to other languages.

A summary of this work is reported in [Amt92].
\end{abstract}




\section{Introduction}

The unfold/fold framework for program transformation dates bank to (at least) [BD77] and has since been the subject of much interest, primarily aimed at making the process of finding "eureka"-definitions more systematic, e.g. [Wad90], [NN90], [PP91b]. Also supercompilation [Tur86] can be seen as a variant over the concept.

A major problem with the technique is that one, due to "too much folding", may risk that the program resulting from transformation (the target program) loops while the original (the source program) does not. A classical example is the following, expressed in a logic language: suppose we have a source program containing the clauses

$p(X) \leftarrow q(X) ; q(a) \leftarrow \square$

Here the query $p(X)$ will succeed with answer substitution $\{X \rightarrow a\}$. However, if one folds the first clause of the program against itself, the target program will contain the clause

$p(X) \leftarrow p(X)$

and now the query $p(X)$ will loop (i.e. neither succeed nor fail).

By innocent abuse of terminology, we will say that a transformation is partially correct iff each time the target program terminates with some result also the source program terminates and with the same result; whereas we will define total correctness to mean partial correctness together with the condition that if the target program does not terminate then neither does the source program. Whether a transformation process itself terminates is beyond the scope of this paper, but e.g. [Wad90], [PP91b] address this problem for certain transformation strategies.

Several ways to guarantee total correctness have been proposed in the literature, e.g. [TS84], [KK90], [Sek91], [Kot85], [GS91], [PP91a]. They all work by putting forward some restrictions on the types of foldings allowed. For a more detailed description (and comparison with our appreach), see section 3 .

The purpose of this paper is to present a model for unfold/fold-transformations which enables one to express conditions which are provably sufficient for total correctness. We want the model to include (most of) the results 
from the literature as special cases (after the frameworks in question have been encoded into our framework); and we want the model to have a clean algebraic structure.

Our framework is primarily aimed at modeling logic programming - even though the machinery differs from the one usually used when treating logic languages, as done in e.g. [Llo84], [Søn89]. However, we believe that the main ideas can be carried over to other types of languages as well.

The meaning of programs will be defined in terms of a transition semantics (cf. [Plo81]). The reason for this is that we feel this is more appropriate for capturing the essence of unfolding and folding: unfolding corresponds to a transition being made in the "right" direction; folding corresponds to a transition being made in the "wrong" direction. By using a denotational approach, this cannot be expressed directly. We believe that the reason why conditions for unfold/fold transformations to be termination preserving apparently is a more hot topic in the logic programming community than in the functional community is that in the former operational semantics (typically derivation trees) has a more respectable status than in the latter.

\subsection{An overview of this paper}

The aim of section 2 will be to give the reader a flavor of the main features of our model. In particular.

- in section 2.1 we introduce the concept of multilevel transition systems, a way to model the relationship between evaluating the source program, transforming the source program and evaluating the target program.

- in section 2.2 we introduce the concept of $U$-mirrors, a representation of (the control part of) an unfold/fold transformation which facilitates reasoning about preservation of termination properties.

- in section 2.3 we focus upon the data aspect, which is usually modeled by means of substitutions - we will propose an alternative approach.

- in section 2.4 we discuss when it is permissible to fold against a given clause - not wrt. total correctness, but wrt. partial correctness.

- in section 2.5 we discuss how to ensure total correctness. Various evaluation strategies are considered. 
- in section 2.6 we discuss how to extend the model such that it is able to represent the whole search tree and not only a single branch.

Section 2 will be rather informal, based on examples and intuition. All concepts introduced will be formally defined and all theorems will be proved in the subsequent sections. Section 3 compares with related work.

In section 4, the basic machinery is set up, e.g. concerning configurations transitions and U-mirrors. In section 5, a multilevel transition system is defined. In section 6 , we state and prove various theorems concerning sufficient conditions for total correctness. In section 7 the whole story is repeated, transitions now representing search trees instead of single branches - here some proofs will appeal rather heavily to intuition, but of course these may be formalized at the expense of decreased clarity.

First, however, we give a "realistic" example of the unfold/fold technique:

Example 1.1 Consider the following source program, written in a logic language:

$f([],[]) \leftarrow$

$f([N \mid U],[\mathrm{s}(N) \mid V]) \leftarrow f(U, V)$

$g(X, Z) \leftarrow f(X, Y), f(Y, Z)$

Operationally, $f$ adds one to each element in a list of unary numbers. Thus $g$ will traverse its input list $X$ twice. Our aim will be to make a target program where $g$ only traverses its input list once: first consider the configuration $g([], Z)$. This can be unfolded into the configuration $f([], Y), f(Y, Z)$. By unfolding the first $f, Y$ gets bound to [] and we arrive at the configuration $f([], Z)$. Now this $f$ can be unfolded, binding $Z$ to []. We are thus able to let the target program contain the rule

$g([],[]) \leftarrow$

Next consider the configuration $g([N \mid X], Z)$. This can be unfolded into $f([N \mid X], Y), f(Y, Z)$. By unfolding the first $f, Y$ gets bound to $[\mathbf{s}(N) \mid Y 1]$ and we get the configuration $f(X, Y 1), f([\mathrm{~s}(N) \mid Y 1], Z)$. By unfolding the second $f, Z$ gets bound to $[\mathrm{s}(\mathrm{s}(N)) \mid Z 1]$ an we arrive at the configuration $f(X, Y 1), f(Y 1, Z 1)$. As $Y 1$ is a new unbound variable, this can now be folded back into the configuration $g(X, Z 1)$ We are thus able to let the target program contain the rule

$g([N \mid X],[\mathrm{s}(\mathrm{s}(N)) \mid Z 1]) \leftarrow g(X, Z 1)$ 
Now consider the "query" $g([0,0], Z)$. If the target program is used "solve" this query, it is first rewritten into $g([0], Z 1)$ binding $Z$ to $[\mathrm{s}(\mathrm{s}(0)) \mid Z 1)$; then rewritten into $g([], Z 2)$ binding $Z 1$ to $[\mathrm{s}(\mathrm{s}(0)) \mid Z 2)$ and finally rewritten into the empty configuration, binding $Z 2$ to []. Thus the query is solved using three inference steps, and $Z$ has been bound to $[\mathrm{s}(\mathrm{s}(0)), \mathrm{s}(\mathrm{s}(0))]$.

It is easily seen that the same query, $g([0,0], Z)$, also can be solved with the same binding to $Z$ by using the source program - but then seven inference steps are needed.

\section{An outline of the theory}

\subsection{Modeling the transformation process}

The key idea is to model computation as transitions between configurations, and to model a program as a collection of distinguished transitions to be called rules. We have a hierarchy as follows:

1. The source program is represented as rules at level 0 . That $t$ is a rule at level 0 is written $t \in \mathcal{R} \mathcal{U}_{0}$.

2. As soon as the rules at level 0 have been given a series of other entities will be fixed:

- the set of level 1 unfolding steps. That $t$ is a level 1 unfolding step from $B$ to $B^{\prime}$ intuitively means that $B^{\prime}$ can be derived by unfolding one of the atoms in $B$, using a rule in $\mathcal{R} \mathcal{U}_{0}$.

- the set of level 1 folding steps. That $t$ is a level 1 folding step from $B$ to $B^{\prime}$ intuitively means that $B^{\prime}$ can be derived from $B$ by performing one folding step, using a rule in $\mathcal{R} \mathcal{U}_{0}$.

- the set of level 1 unfoldings. That $t$ is a level 1 unfolding from $B$ to $B^{\prime}$ means that $B^{\prime}$ can be derived by from $B$ by a sequence of unfoldings, using rules in $\mathcal{R} \mathcal{U}_{0}$.

- the set of level 1 foldings. That $t$ is a level 1 folding from $B$ to $B^{\prime}$ means that $B^{\prime}$ can be derived from $B$ by a sequence of foldings, using rules in $\mathcal{R} \mathcal{U}_{0}$.

- the set of level 1 transitions. That $t$ is a level 1 transition from $B$ to $B^{\prime}$ means that $B^{\prime}$ can be derived by from $B$ by a sequence of unfoldings and foldings, using rules in $\mathcal{R} \mathcal{U}_{0}$. 
Level 1 unfoldings model standard evaluation of the source program; whereas level 1 transitions model transformation ("symbolic evaluation") of the source program.

3. Among all level 1 transitions, some are chosen to be rules at level 1 that $t$ is a rule at level 1 is written $t \in \mathcal{R} \mathcal{U}_{1}$. These rules represent the target program.

4. As soon as the rules at level 1 have been given, the level 2 unfolding steps and the level 2 unfoldings are fixed. That $t$ is a level 2 unfolding step (unfolding) from $B$ to $B^{\prime}$ means that $B^{\prime}$ can be derived from $B$ by a (sequence of) unfoldings, using rules in $\mathcal{R} \mathcal{U}_{1}$.

Level 2 unfoldings thus model (standard) evaluation of the target program.

For inference rules determining the set of level 1 unfoldings etc, see section 5. Not surprisingly, it will hold that if $t$ is a level 2 unfolding it also is a level 1 transition.

A key point of our approach is that "standard evaluation" (the level 1 unfoldings) is a special case of "symbolic evaluation" (the level 1 transitions) - this greatly facilitates reasoning about the properties of the target program. This lack of distinction between standard evaluation and symbolic evaluation comes almost for free in a logic language, but also in the functional world one gains from viewing the latter as a generalization of the former [DP88]. However, an important difference between standard and symbolic evaluation is that during symbolic evaluation any atom in the goal sequence may be unfolded, whereas during standard evaluation one for efficiency reasons often chooses a fixed strategy, typically the strategy always to unfold the leftmost goal - this strategy will be denoted $\mathcal{L} \mathcal{R}$.

\subsection{Modeling control}

A transition $t$ from $B$ to $B^{\prime}$ will be represented by means of $U$-mirrors: intuitively speaking, a U-mirror is a triple $\left(f, f^{\prime}, B^{\prime \prime}\right)$ where $f$ is a $U$-forest describing how to get from $B$ to $B^{\prime \prime}$ by means of unfoldings using level 0 rules; and $f^{\prime}$ is a U-forest describing how to get from $B^{\prime}$ to $B^{\prime \prime}$ by means of unfoldings using level 0 rules. Thus, if $t$ is a level 1 unfolding $f^{\prime}$ will be trivial; and if $t$ is a level 1 folding $f$ will be trivial. U-mirrors will be treated in depth in section 4.2 . 

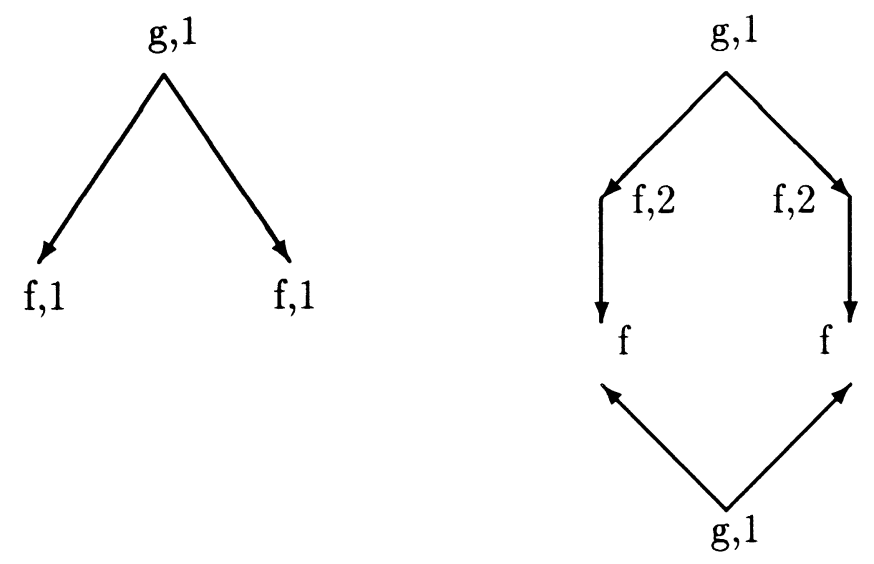

Figure 1: Two U-mirrors

In figure 1 is depicted the U-mirrors corresponding to the level 1 rules (1) and (2) from example 1.1. That the two leaves of the first U-mirror are labeled " $f, 1$ " is because the two occurrences of $f$ were unfolded when deriving the rule, in both cases using the first level 0 rule for $f$. That two internal nodes in the second U-mirror are labeled " $f, 2$ " is because the two occurrences of $f$ were unfolded when deriving the rule, in both cases using the second level 0 rule for $f$.

Now suppose the target program loops on some configuration $B$, i.e. there exists an infinite sequence of level 2 unfolding steps from $B$. As each such unfolding step is represented by the second U-mirror in figure 1, it is easily seen - as a folding into $g$ is "canceled" by a subsequent unfolding of $g$ - that this means that from $B$ there exists an infinite sequence of level 1 unfolding steps, where the first step unfolds $g$ and the remaining steps unfold $f$. This informally shows the total correctness of the transformation.

Of course, it is also possible to argue for total correctness by observing that the first argument to $g$ gets "smaller" for each inference step (assuming that $g$ is called with a first argument which is fully instantiated). However, the virtue of the abovementioned way of reasoning is that it only depends on the syntactic structure of the transformation process. 


\subsection{Modeling data}

A configuration is a sequence of goals together with some information about which values the variables in the goals can assume. One usually represents this information as a substitution, cf. [Llo84]. As substitutions 7are hard to reason about from an algebraic point of view (even though e.g. [Søn89] and [Pal89] show that certain sets of substitutions carry some structure), in particular one has to be careful about renaming, we will represent the information as a family of sets of ground values, to be called an information family. For a full account of configurations and operations on these, see section 4.1.

We will now briefly sketch how the standard framework translates into our framework. In the following, assume that $\mathcal{D}$ is a universal data domain. ${ }^{1}$

As an example take the goal sequence $(p(X), q(Y), r(Z))$ together with the substitution $\{X \rightarrow f(Y), Z \rightarrow a\}$. This in our framework could be represented as the goal sequence $(p, q, r)$ together with the $\mathcal{D}$-indexed family where the $d$ 'th element is the singleton set $\{(f(d), d, a)\}$ - on the other hand, one might also use the family consisting of one element only, namely the set $\{(f(d), d, a) \mid d \in \mathcal{D}\}$. The latter representation will be needed for dealing with "variables occurring on the right hand side but not on the left hand side"; we will elaborate on this issue in section 2.4.

We now return to example 1.1. The level 0 rule $f([],[]) \leftarrow \square$ is represented as a transition from $B_{1}=\left([f], Q_{1}\right)$ to $B_{1}^{\prime}=\left([], Q_{1}^{\prime}\right)$, where $Q_{1}$ and $Q_{1}^{\prime}$ are $\mathcal{D} \times$ $\mathcal{D}$-indexed families with $Q_{1}\left(d_{1}, d_{2}\right)=\left\{\left(d_{1}, d_{2}\right)\right\}, Q_{1}^{\prime}([],[])=\{()\}, Q_{1}^{\prime}\left(d_{1}, d_{2}\right)=$ $\emptyset$ if $d_{1} \neq[]$ or $d_{2} \neq[]$.

The level 0 rule $f([N \mid U],[\mathbf{s}(N) \mid V) \leftarrow f(U, V)$ is represented as a transition from $B_{2}=\left([f], Q_{2}\right)$ to $B_{2}^{\prime}=\left([f], Q_{2}^{\prime}\right)$ where the $\mathcal{D} \times \mathcal{D}$-indexed information families $Q_{2}$ and $Q_{2}^{\prime}$ are given by $Q_{2}\left(d_{1}, d_{2}\right)=\left\{\left(d_{1}, d_{2}\right)\right\}, Q_{2}^{\prime}\left(\left[d_{n} \mid\right.\right.$ $\left.\left.d_{u}\right],\left[\mathbf{s}\left(d_{n}\right) \mid d_{v}\right]\right)=\left\{\left(d_{u}, d_{v}\right)\right\}$ and $Q_{2}^{\prime}\left(d_{1}, d_{2}\right)=\emptyset$ if $\left(d_{1}, d_{2}\right)$ is not of the form above.

In the standard framework, the query $f([0], Z)$ is solved yielding an answer substitution where $Z$ is bound to $[\mathrm{s}(0)]$. Now consider how this works in our framework. There the query $f([0], Z)$ is represented as the configuration $B=([f], Q)$ where the $\mathcal{D}$-indexed information family $Q$ is given by $Q(d)=\{([0], d)\}$. Now consider the mapping $s$ from $\mathcal{D}$ to $\mathcal{P}(\mathcal{D} \times \mathcal{D})$ given

\footnotetext{
${ }^{1}$ We will impose no requirements on the structure of this set; in our examples, however, we shall assume the elements of $\mathcal{D}$ to be PROLOG ground terms, i.e. terms built inductively from some set of functors (constants just being zero-arity functors).
} 
by $s(d)=Q(d)$. Then for all $d$ it will trivially hold that

$$
Q(d)=\bigcup_{\left(d_{1}, d_{2}\right) \in s(d)} Q_{2}\left(d_{1}, d_{2}\right)
$$

The existence of this $s$ shows that $B$ is an instance of $B_{2}$, to be written $B=\mathcal{I}_{s}\left(B_{2}\right)$. Then there will be a level 1 unfolding step from $B$ to $\mathcal{I}_{s}\left(B_{2}^{\prime}\right)$, to be denoted $B^{\prime}$. $B^{\prime}=\left([f], Q^{\prime}\right)$ where $Q^{\prime}$ is a $\mathcal{D}$-indexed family given by

$$
Q^{\prime}(d)=\bigcup_{\left(d_{1}, d_{2}\right) \in s(d)} Q_{2}^{\prime}\left(d_{1}, d_{2}\right)
$$

That is, $Q^{\prime}\left(\left[\mathbf{s}(0) \mid d_{v}\right]\right)=\left\{\left([], d_{v}\right)\right\}$ and $Q^{\prime}(d)=\emptyset$ otherwise.

Next consider the mapping $s^{\prime}$ from $\mathcal{D}$ to $\mathcal{P}(\mathcal{D} \times \mathcal{D})$ given by $s^{\prime}(d)=Q^{\prime}(d)$. Then for all $d$ it will trivially hold that

$Q^{\prime}(d)=\bigcup_{\left(d_{1}, d_{2}\right) \in s^{\prime}(d)} Q_{1}\left(d_{1}, d_{2}\right)$

This means that $B^{\prime}=\mathcal{I}_{s^{\prime}}\left(B_{1}\right)$. Then there will be a level 1 unfolding step from $B^{\prime}$ to $\mathcal{I}_{s^{\prime}}\left(B_{1}^{\prime}\right)$, to be denotes $B^{\prime \prime} . B^{\prime \prime}=\left([], Q^{\prime \prime}\right)$ where $Q^{\prime \prime}$ is a $\mathcal{D}$-indexed family given by

$$
Q^{\prime \prime}(d)=\bigcup_{\left(d_{1}, d_{2}\right) \in s^{\prime}(d)} Q_{1}^{\prime}\left(d_{1}, d_{2}\right)
$$

That is, $Q^{\prime \prime}\left(\left[\mathrm{s}(0) \mid d_{v}\right)=Q_{1}\left([], d_{v}\right)\right.$ and $Q^{\prime \prime}(d)=\emptyset$ otherwise, i.e. $Q^{\prime \prime}([\mathrm{s}(0)])=$ $\{()\}$ and $Q^{\prime \prime}(d)=\emptyset$ otherwise. As $Q^{\prime \prime}(d) \neq \emptyset$ iff $d=[\mathbf{s}(0)]$, this corresponds to $Z$ being bound to $[\mathrm{s}(0)]$ in the standard model.

Of course, also $B^{\prime}=\mathcal{I}_{s^{\prime}}\left(B_{2}\right)$. So there also is a level 1 unfolding from $B^{\prime}$ to $\mathcal{I}_{s^{\prime}}\left(B_{2}^{\prime}\right)=B^{\prime \prime \prime}$, where $B^{\prime \prime \prime}=\left([f], Q^{\prime \prime \prime}\right)$. However, it is easily seen that $Q^{\prime \prime \prime}(d)=\emptyset$ for all $d$ - we say that $B^{\prime \prime \prime}$ is a failure configuration. Thus the transition from $B^{\prime}$ to $B^{\prime \prime \prime}$ represents a failure branch.

In our examples we will, for ease of exposition, often switch back and forth between the standard model and our model when it is the control aspect which has our primary interest.

\subsection{Modeling folding}

Let some predicate symbol $G$ be given, and let the level 0 rules for $G$ be of

form $\left\{t_{i} \mid i \in I\right\}$, each $t_{i}$ going from $B$ to $B_{i}$. Here $B$ contains goal sequence 
$G$ and $K$-indexed information family $Q$, and each $B_{i}$ contains $K$-indexed information family $Q_{i}$.

Given $i \in I$. Now suppose $s$, a mapping from $K^{\prime}$ to $\mathcal{P}(K)$, is such that

1. with $Q_{i}^{\prime}$ the $K^{\prime}$-indexed information family of $\mathcal{I}_{s}\left(B_{i}\right), Q_{i}^{\prime}\left(k^{\prime}\right) \neq \emptyset$ for all $k \in K^{\prime}$

2. $\mathcal{I}_{s}\left(B_{i^{\prime}}\right)$ is failure for $i^{\prime} \neq i$

3. $B_{i}$ consists of a non-empty goal sequence.

Then it will be possible to make a level 1 folding step from $\mathcal{I}_{s}\left(B_{i}\right)$ to $\mathcal{I}_{s}\left(B_{i}\right)$, cf. the definition in section 5.4.

The rationales for the above requirements are as follows:

1. It must not be possible to make a folding step from a failure configuration into a non-failure configuration. To see whys consider the two program clauses:

$p(a) \leftarrow q(a) ; q(X) \leftarrow q(X)$

Starting with the configuration $p(X)$, one may consider unfolding it into $q(a)$, then unfold it once more into $\mathrm{q}(\mathrm{a})$, and finally (erroneously!) fold back into $p(X)$ - thus deriving the target program $p(X) \leftarrow p(X)$. As two unfoldings and only one folding is made, the reasoning in section 2.2 may tempt us to believe that this transformation preserves termination properties. However, e.g. the goal $p(b)$ loops at level 2 (i.e. when using the target program); while it fails when evaluated at level 1. This is because the infinite sequence of level 2 unfolding steps $p(b) \rightarrow p(b) \rightarrow \ldots$ corresponds to the sequence of level 1 unfold/fold steps where $p(b)$ is unfolded into failure which then is unfolded into failure which then is folded back to $p(b)$ etc.

To see why the folding from $q(a)$ to $p(X)$ is not a level 1 folding step in our model, notice that the clause $p(a) \leftarrow q(a)$ is represented as a transition from $B$ to $B_{1}$, containing $\mathcal{D}$-indexed information families $Q$ and $Q_{1}$ respectively. Here $Q(d)=\{d\}$ for all $d \in \mathcal{D}$, while $Q_{1}(a)=\{a\}$ and $Q_{1}(d)=\emptyset$ for $d \neq a$.

2. This in the standard framework is modeled by the requirement that only one clause defining the predicate folded against should match: if we have two program clauses 
$p \leftarrow q ; p \leftarrow r$

it must not be possible to fold $r$ into $p$ and then unfold into $q$ - this would destroy semantics.

3. If there is a source program clause $p \leftarrow \square$, it should not be possible to fold e.g. $q$ into $q, p$. Such foldings never occur in practice, and it is convenient to exclude them: otherwise we above could derive the target program $p \leftarrow p$, and then $p$ would loop at level 2 but as the corresponding level 1 transition unfolds $p$ into [] which then is folded back into $p$ etc, $p$ does not loop at level 1 .

When folding in a logic language, one has to be careful when folding against a clause containing variables not occurring in the head. This is a problem to which some incorrect solutions have been proposed in the literature (and yet proved correct!), for a survey see [GS91].

In our framework, this problem is solved "for free": consider e.g. the clause from example $1.1 g(X, Z) \leftarrow f(X, Y), f(Y, Z)$ which is represented as a transition from $B=\left([g],\left\{\left\{\left(d_{1}, d_{2}\right)\right\} \mid d_{1}, d_{2} \in \mathcal{D}\right\}\right)$ to $B^{\prime}=\left([f, f], Q^{\prime}\right)$ where the $\mathcal{D} \times \mathcal{D}$-indexed information family $Q^{\prime}$ is given by $Q^{\prime}\left(d_{1}, d_{2}\right)=$ $\left\{\left(d_{1}, d, d, d_{2}\right) \mid d \in \mathcal{D}\right\}$. Now suppose $B^{\prime \prime}=\mathcal{I}_{s}\left(B^{\prime}\right)$ for some $s$, with $B^{\prime \prime}=$ $\left([f, f], Q^{\prime \prime}\right)$. For any $k$ (in the domain of $s$ ) we have

$$
Q^{\prime \prime}(k)=\bigcup_{\left(d_{1}, d_{2}\right) \in s(k)} Q^{\prime}\left(d_{1}, d_{2}\right)
$$

so if $Q^{\prime \prime}(k)$ contains an element of the form $\left(d_{1}, d, d, d_{2}\right)$ then for all $d^{\prime} \in$ $\mathcal{D}$ also $Q^{\prime \prime}(k)$ contains $\left(d_{1}, d^{\prime}, d^{\prime}, d_{2}\right)$. This means - switching back to the standard framework - that if $Y$ is instantiated in $f(X, Y), f(Y, Z)$ then this configuration cannot be written on the form $\mathcal{I}_{s}\left(B^{\prime}\right)$.

On the other hand, if $Y$ is uninstantiated and a "new variable" then it is possible to fold back into $g$, as done when deriving rule (2) in example 1.1. Let us do so, within our framework: we start with the configuration $B_{1}=\left([g], Q_{1}\right)$ where the $\mathcal{D} \times \mathcal{D} \times \mathcal{D}$-indexed information family $Q_{1}$ is given by

$Q_{1}\left(d_{1}, d_{2}, d_{3}\right)=\left\{\left(\left[d_{1} \mid d_{2}\right], d_{3}\right)\right\}$

By unfolding $g$, we get the configuration $B_{2}=\left([f, f], Q_{2}\right)$ where the $\mathcal{D}^{3}$ indexed information family $Q_{2}$ is given by

$Q_{2}\left(d_{1}, d_{2}, d_{3}\right)=\left\{\left(\left[d_{1} \mid d_{2}\right], d, d, d_{3}\right) \mid d \in \mathcal{D}\right\}$ 
Now the first $f$ is unfolded, and we get the configuration ${ }^{2} B_{3}=\left([f, f], Q_{2}\right)$ where the $\mathcal{D}^{3}$-indexed information family $Q_{3}$ is given by

$Q_{3}\left(d_{1}, d_{2}, d_{3}\right)=\left\{\left(d_{2}, d^{\prime},\left[\mathbf{s}\left(d_{1}\right) \mid d^{\prime}\right], d_{3}\right) \mid d^{\prime} \in \mathcal{D}\right\}$

By unfolding the second $f$ we get the configuration $B_{4}=\left([f, f], Q_{4}\right)$ where the $\mathcal{D}^{3}$-indexed information family $Q_{4}$ is given by

$Q_{4}\left(d_{1}, d_{2},\left[\mathbf{s}\left(\mathbf{s}\left(d_{1}\right)\right) \mid d_{3}^{\prime}\right]\right)=\left\{\left(d_{2}, d^{\prime}, d^{\prime}, d_{3}^{\prime}\right) \mid d^{\prime} \in \mathcal{D}\right\}, Q_{4}\left(d_{1}, d_{2}, d_{3}\right)=\emptyset$ otherwise

Now define $s$, a mapping from $\mathcal{D}^{3}$ to $\mathcal{P}\left(\mathcal{D}^{2}\right)$, as follows:

$s\left(d_{1}, d_{2},\left[\mathbf{s}\left(\mathbf{s}\left(d_{1}\right)\right) \mid d_{3}^{\prime}\right]\right)=\left\{\left(d_{2}, d_{3}^{\prime}\right)\right\}, s\left(d_{1}, d_{2}, d_{3}\right)=\emptyset$ otherwise

Then we have $B_{4}=\mathcal{I}_{s}\left(B^{\prime}\right)$, as

$$
Q_{4}\left(d_{1}, d_{2}, d_{3}\right)=\bigcup_{\left(d_{1}^{\prime}, d_{2}^{\prime}\right) \in s\left(d_{1}, d_{2}, d_{3}\right)} Q^{\prime}\left(d_{1}^{\prime}, d_{2}^{\prime}\right)
$$

So then we can fold $B_{4}$ into $B_{5}=\mathcal{I}_{s}(B)$, with $B_{5}=\left([g], Q_{5}\right)$ where the $\mathcal{D}^{3}$-indexed information family $Q_{5}$ is given by $Q_{5}=s$, ie.

$Q_{5}\left(d_{1}, d_{2},\left[\mathbf{s}\left(\mathbf{s}\left(d_{1}\right)\right) \mid d_{3}^{\prime}\right]\right)=\left\{\left(d_{2}, d_{3}^{\prime}\right)\right\}, Q_{5}\left(d_{1}, d_{2}, d_{3}\right)=\emptyset$ otherwise

The level 1 transition from $B_{1}$ to $B_{5}$, translated into the standard framework, is just rule (2).

\subsection{Conditions for total correctness}

Consider the program

$$
\begin{aligned}
& E(a) \leftarrow A \\
& E(b) \leftarrow B \\
& E(X: Y) \leftarrow E(X), E(Y) \\
& A \leftarrow B, B
\end{aligned}
$$

$\cdots$

Starting with $E(a)$, we can unfold this into $A$ and further into $B, B$. This can be folded bank into $B, E(b)$ into $E(b), E(b)$ and finally folded back into

\footnotetext{
${ }^{2}$ It will be a good exercise for the reader to check this, after having read section 5.2.
} 
$E(b: b)$, yielding a target program

$E(a) \leftarrow E(b: b)$

As two unfolding steps and three folding steps have been made, the reasoning technique from section 2.2 cannot be used to show total correctness of the transformation. However, we can argue that the clause above represents some progress in the computation process, as $A$ is unfolded into $B, B$ but never folded back. This can be formalized by assigning weights (non-negative numbers) to the arcs in the U-mirrors representing a transition, such that the weight of an arc is a function of the predicate symbol being unfolded. ${ }^{3}$ We can now define the weight of a path in a U-mirror $\left(f, f^{\prime}\right)$ as the sum of the weights encountered when walking along the path, where the weights of arcs in $f^{\prime}$ are negated before contributing to the summation.

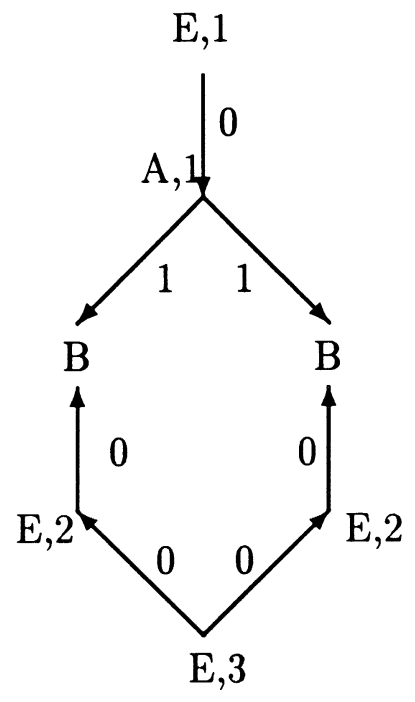

Figure 2: A U-mirror with weights

By assigning arcs from $E$ weight 0 and arcs from $A$ weight 1 , the target program clause above is represented by the U-mirror depicted in figure 2 . We see that all paths have weights 1 - but if we had assigned $E$ weight 2 all paths would have weight -1 .

Informally, a fair strategy sooner or later unfolds any goal. Then we have

\footnotetext{
${ }^{3}$ Actually, the weight may also depend on which rule is used and which conjunct the arc represents.
} 
Condition 2.1 Suppose it for all level 1 rules holds that all the paths in the corresponding $\mathrm{U}$-mirror have weight $\geq 1$. Suppose $B$ loops at level 2 by a fair strategy. Then $B$ loops at level 1 by a fair strategy too. (This is theorem 6.2.)

Thus we have found a condition for a transformation to be total correct wrt. a fair evaluation strategy. Concerning total correctness wrt. the $\mathcal{L} \mathcal{R}$ strategy, we have

Condition 2.2 Suppose it for all level 1 rules holds that the leftmost path in the corresponding U-mirror has weight $\geq 1$. Suppose $B$ loops at level 2 by the $\mathcal{L} \mathcal{R}$ strategy. Then $B$ loops at level 1 by the $\mathcal{L} \mathcal{R}$ strategy too. (This is theorem 6.3.)

On the other hand, if the transformation does some non- $\mathcal{L} \mathcal{R}$ steps it may happen that the domain of termination is increased. To see this, consider the source program

$p(X) \leftarrow q(X), r(X) ; q(a) \leftarrow q(a) ; r(b) \leftarrow$

Starting with the configuration $p(X)$, this can be unfolded into $q(X), r(X)$ and then by a non- $\mathcal{L} \mathcal{R}$ unfolding into $q(b)$, yielding the target program

$p(b) \leftarrow q(b)$

Now $p(X)$ terminates (and fails) at level 2 by any strategy, while $p(X)$ loops at level 1 by the $\mathcal{L} \mathcal{R}$ strategy.

The same source program shows that it may happen that a transformation is total correct wrt. the $\mathcal{L} \mathcal{R}$ strategy but not wrt. a fair strategy: again starting with the configuration $p(\mathrm{X})$ we unfold this into $q(X), r(X)$ and then we unfold the leftmost atom yielding $q(a), r(a)$. This can be folded back into $p(a)$, yielding the target program

$p(a) \leftarrow p(a)$

It is easily seen that this transformation is total correct wrt. the $\mathcal{L} \mathcal{R}$ strategy - $p(t)$ loops at level 2 (by the $\mathcal{L} \mathcal{R}$ strategy) iff $t$ can be unified with $a$ iff $p(t)$ loops at level 1 by the $\mathcal{L} \mathcal{R}$ strategy. This is as predicted by condition 2.2 , since it is possible to assign weights in a way (e.g. 1 to $q$ and 0 to $p$ ) such that the leftmost path of the U-mirror corresponding to this transformation has weight $\geq 1$.

On the other hand, $p(X)$ loops at level 2 (by any strategy) but terminates at level 1 by a fair strategy. Thus the transformation is not total correct wrt. 
a fair strategy.

Having defined the weight of a U-mirror $\left(f, f^{\prime}\right)$ as the sum of the weights occurring in it, the weights occurring in $f^{\prime}$ negated, we can formulate a - less useful - condition:

Condition 2.3 Suppose it for all level 1 rules holds that the corresponding U-mirror has weight $\geq 1$. Suppose $B$ loops at level 2 by some strategy. Then $B$ also loops at level 1 , by some strategy. (This is theorem 6.1 .)

This condition is not enough to guarantee total correctness (neither wrt. fair nor $\mathcal{L} \mathcal{R}$ semantics): consider the source program $p(X) \leftarrow r(X), q(X)$; $q(a) \leftarrow q(a) ; r(b) \leftarrow \square$. By unfolding $p$; unfolding $q$ and finally folding into $p$ we get the level 1 rule $p(a) \leftarrow p(a)$. If $q$ is assigned weight $\geq 1$, the corresponding $\mathrm{U}$-mirror will have weight $\geq 1$. Now e.g. $p(a)$ loops at level 2 by any strategy, but fails at level 1 by a fair strategy as well as by the $\mathcal{L} \mathcal{R}$ strategy.

\subsection{Modeling the full search tree}

So far a transition - for ease of exposition - only represents a single branch of the search tree, the transition system thus being non-confluent. In order to model the full search tree, configurations have to be multisets of "old" configurations (now to be called basic configwations). There are two reasons for working with multisets and not with sequences (i.e. not to order the branches), a pragmatic and a mathematical one:

- it is rather easy to implement or-parallelism [Gre87], as no communication has to occur between the branches. On the other hand, andparallelism [Gre87] is much harder to implement due to the need for sharing of data, hence most implementations employ the $\mathcal{L} \mathcal{R}$ strategy.

- If we use sequences, the Church-Rosser property will be lost. To see this, consider the program

$a \leftarrow b ; a \leftarrow c ; d \leftarrow e ; d \leftarrow f ;$

Now consider the goal $(a, d)$ By first unfolding a and then unfolding $d$ we first get $(b, d) ;(c, d)$ and then $B_{1}=(b, e) ;(b, f) ;(c, e) ;(c, f)$. By first unfolding $d$ and then unfolding $a$ we first get $(a, e) ;(a, f)$ and then $B_{2}=(b, e) ;(c, e) ;(b, f) ;(c, f)$. In [PP91a] one wants to distinguish 
between $B_{1}$ and $B_{2}$, and therefore unfolding of the leftmost atom only is allowed (unless extra conditions are satisfied.)

A configuration is said to be in normal form if all the basic configurations belonging to it are non-failure and with an empty goal sequence. Due to the Church-Rosser property, it then for a (basic) configuration $B$ makes sense to define $\llbracket B \rrbracket_{1}$ as follows: if there exists a $C$ in normal form and a level 1 unfolding from $B$ to $C, \llbracket B \rrbracket_{1}=C$. Otherwise, $\llbracket B \rrbracket_{1}=\perp$. In a similar vein, one can define $\llbracket B \rrbracket_{2}$. By restricting the level 1 (2) unfoldings in question to be $\mathcal{L} \mathcal{R}$, one can define $\llbracket B \rrbracket_{1}^{L}\left(\llbracket B \rrbracket_{2}^{L}\right)$. Now condition 2.1 and 2.2 can be restated (a rule may now be represented by several U-mirrors):

Condition 2.4 Suppose that for all level 1 rules, represented by U-mirrors $m_{1} \ldots m_{k}$, it holds for all $m_{i}$ that all paths in $m_{i}$ have weight $\geq 1$. Then for all $B, \llbracket B \rrbracket_{2}=\llbracket B \rrbracket_{1}$.

Condition 2.5 Suppose that for all level 1 rules, represented by U-mirrors $m_{1} \ldots m_{k}$, it holds for all $m_{i}$ that the leftmost path in $m_{i}$ has weight $\geq 1$. Then for all $B, \llbracket B \rrbracket_{2} \geq \llbracket B \rrbracket_{1}$ (notice that the domain of termination may be increased, as shown in section 2.5).

For a more detailed treatment and for proofs, see section 7 .

In one way, the expressive power is enhanced by working with the full search tree: we can fold a configuration containing several basic configurations back into a single basic configuration - resembling the process of converting a NFA into a DFA. As an example of this, consider the program

$a b([]) \leftarrow \square ; a b([a \mid X]) \leftarrow a b(X) ; a b([b \mid X]) \leftarrow a b(X)$

$b c([]) \leftarrow \square ; b c([b \mid X]) \leftarrow b c(X) ; b c([c \mid X]) \leftarrow b c(X)$

$a b c(X) \leftarrow a b(X) ; a b c(X) \leftarrow b c(X)$

Now consider the configuration $a b c([])$. This is unfolded into $a b([]) ; b c([])$ which by two unfoldings yield $\square ; \square$. The configuration $a b c([a \mid X])$ is unfolded into $a b([a \mid X)] ; b c([a \mid X])$ which by two unfoldings yield $a b(X)$ (as the second basic configuration is unfolded into failure). In a similar vein, the configuration $a b c([c \mid X])$ is unfolded into $b c(X)$. 
The interesting case is where we start with the configuration $a b c([b \mid X])$. Then we unfold into $a b([b \mid X]) ; b c([b \mid X])$, two more unfoldings yield $a b(X) ; b c(X)$ and now this can be folded back into $a b c(X)$. We have thus derived five new rules for $a b c$ :

$a b c([]) \leftarrow \square ; a b c([]) \leftarrow \square ;$

$a b c([a \mid X]) \leftarrow a b(X) ; a b c([c \mid X]) \leftarrow b c(X)$

$a b c([b \mid X]) \leftarrow a b c(X) ;$

To the latter rule correspond two U-mirrors, depicted in figure 3 .

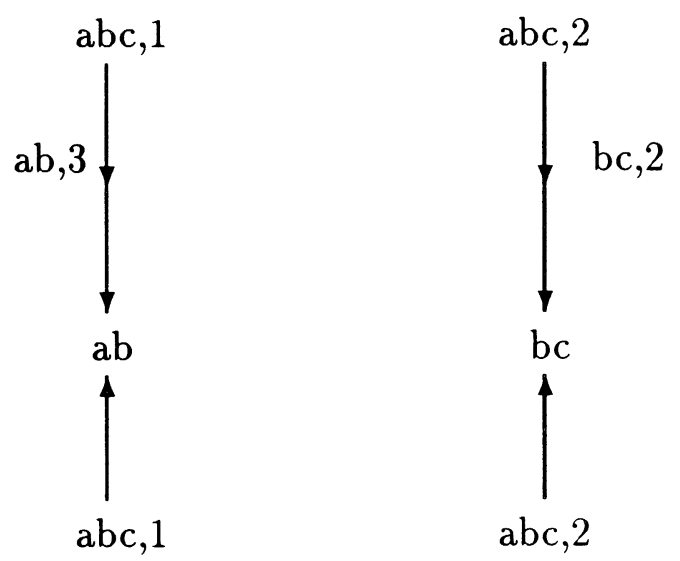

Figure 3: The two U-mirrors for $a b c([b \mid X]) \leftarrow a b c(X)$

\section{Related work}

In the literature on unfold/fold transformations in logic languages transformation typically proceeds in a "step by step fashion"; after a goal in the body of a clause has been unfolded the clause is deleted from the program and replaced by the clause resulting from the unfolding - this is the approach taken in e.g. [GS91], [KK90], [PP91a], [Sek91], [TS84]. As pointed out in [GS91], one by applying this method loses some power - to see this, consider the clause $C=p(f(X)) \leftarrow p(X)$. By our or similar techniques one is able 
to derive the clause $C^{\prime}: p(f(f(f(X)))) \leftarrow p(X)$ but this is impossible by the step-by-step method, since one - after having unfolded $C$ against itself obtaining $p(f(f(X))) \leftarrow p(X)$ - has lost $C$. Aside from being less powerful, we also think that the step-by-step strategy conceptually is much less clean than our approach - a similar view being held in [Tur86].

In the literature, one is typically (contrary to our framework) not allowed to fold against a (direct or indirect) recursive predicate [KK90], [PP91a], [Sek91], [TS84]. This mirrors the view that folding corresponds to abbreviation, a view also held in [Han91].

[TS84] and [KK90] divide the predicates into two classes: the new (corresponding to "eureka-definitions") and old, where folding is allowed against new predicates only. In the body of new predicates as well as in the body of old predicates, only old predicates can occur. Folding is valid in two cases:

- Starting with the definition of an old predicate, $O \leftarrow O_{1} \ldots O_{n}$, one can do zero or more unfoldings of some of the $O_{i}$ 's and then fold some of these back into a new predicate.

- Starting with the definition of a new predicate, $N \leftarrow O_{1} \ldots O_{n}$, one has to do at least one unfolding of some of the $O_{i}$ 's before folding back into a new predicate. ${ }^{4}$

If new predicates are assigned weight 0 and old predicates are assigned weight 1 , this translates into our condition 2.3. As we have seen in section 2.5 this condition is (too) weak, since failing branches may convert to loops.

[Sek91] improves on the above, essentially by coming up with condition 2.1 (still when new predicates have been assigned weight 0 and old predicates weight 1). As now not only the success set but also the failure set is preserved, negation can be handled as well.

[Kot85] treats a functional language (where there apparently is no branching), thus his results are not immediately compatible to ours. The situation is that first a number of unfoldings are made, then some laws are applied (not catered for by our framework), then some foldings are made. It is claimed that folding is safe if the number of unfoldings is greater than the number of

\footnotetext{
${ }^{4}$ Actually, in [TS84] one is allowed to fold even if no unfolding of an $O_{i}$ is made, provided not all the $O_{i}$ 's disappear by the folding. By assigning new predicates a weight equal the number of goals on the right hand side of their definition, and by assigning old predicates a "very large integer" as weight, this translates into our condition 2.3.
} 
foldings. In some sense, this corresponds to assigning all predicates weight 1 in our framework.

[GS91] allows folding against existing clauses (recall clauses are deleted after having been unfolded) only (not allowing a clause to be folded against itself). This greatly limits the applications, since it seems impossible to arrive at recursive definitions of eureka-predicates. On the other hand, it becomes possible to give a relatively simple proof of termination preservation.

In contrast to the authors mentioned so far, [PP91a] impose an order on a sequence of goals, i.e. consider PROLOG's $\mathcal{L} \mathcal{R}$ strategy. The crucial condition on folding is that the leftmost atom has been unfolded. Again by assigning the predicates folded against weight 0 and the others 1 , the essence of this translates into our condition 2.2. A version of condition 2.2 is also stated in [Han91].

\section{Fundamental concepts}

\subsection{Basic configurations}

Assume a finite universe of predicate symbols $U$.

Definition 4.1 A goal sequence $(J, H)$ consists of a totally ordered set $J$, together with a mapping $H$ which to each $j \in J$ assigns a member of $U$.

Often we drop $J$ and just write $H . \quad j<j^{\prime}$ models that $H(j)$ is "to the left" of $H\left(j^{\prime}\right)$.

Definition 4.2 A basic configuration (over $K$ ) is a quadruple $(J, H, K, Q$ ) where $(J, H)$ is a goal sequence, $K$ is a set and $Q$ is a mapping which to each $k \in K$ assigns a member of $\mathcal{P}\left(\prod_{j \in J} \mathcal{D}\right.$ ) (for simplicity, we assume that all predicates have arity 1 ).

A basic configuration is failure if $Q(k)=\emptyset$ for all $k \in K$; and is empty if $J=\emptyset$.

Definition 4.3 Given goal sequence $(J, H)$, we define the canonical basic configuration over $(J, H)$ as follows: $C a_{(J, H)}=\left(J, H, \prod_{j \in J} \mathcal{D}, Q\right)$ where $Q(\vec{d})=\{\vec{d}\}$. 


\section{Specializations}

Definition 4.4 Given basic configurations $B$ and $B^{\prime}$, with $B=(J, H, K, Q)$ and $B^{\prime}=\left(J, H, K^{\prime}, Q^{\prime}\right)$. A specialization from $B$ to $B^{\prime 5}$ is a mapping $s$ from $K$ to $\mathcal{P}\left(K^{\prime}\right)$ such that for all $k \in K$

$$
Q(k)=\bigcup_{k^{\prime} \in s(k)} Q^{\prime}\left(k^{\prime}\right)
$$

We say that $B=\mathcal{I}_{s}\left(B^{\prime}\right)$.

Fact 4.5 Given basic configuration $B=(J, H, K, Q)$. Now there exists one and only one specialization $s$ from $B$ to $C a_{(J, H)}$.

Proof: $s$ will be a specialization iff

$$
Q(k)=\bigcup_{\vec{d} \in s(k)}\{\vec{d}\}=s(k)
$$

\section{Operators on configurations and specializations}

Definition 4.6 If $J_{1}$ and $J_{2}$ are two ordered sets (ordered by $<_{1}$ and $<_{2}$ ), we define $J=J_{1} \& J_{2}$ (ordered by $<$ ) by letting $J$ be the disjoint union of $J_{1}$ and $J_{2}$ by letting $i n_{1}(j)<i n_{1}\left(j^{\prime}\right)$ iff $j<_{1} j^{\prime}$ and $i n_{2}(j)<i n_{2}\left(j^{\prime}\right)$ iff $j<_{2} j^{\prime}$; and by letting $i n_{1}(j)<i n_{2}\left(j^{\prime}\right)$ for all $j \in J_{1}, j^{\prime} \in J_{2}$.

Definition 4.7 Let $\left(J_{1}, H_{1}\right)$ and $\left(J_{2}, H_{2}\right)$ be two goal sequences. We define $\left(J_{1}, H_{1}\right) \&\left(J_{2}, H_{2}\right)(=(J, H))$ as follows: $J=J_{1} \& J_{2} ; H\left(i n_{1}(j)\right)=H_{1}\left(j_{1}\right)$ and $H\left(i n_{2}(j)\right)=H_{2}(j)$.

Definition 4.8 Let $B_{1}=\left(J_{1}, H_{1}, K_{1}, Q_{1}\right)$ and $B_{2}=\left(J_{2}, H_{2}, K_{2}, Q_{2}\right)$ be basic configurations. Then we define $B_{1} \& B_{2}=(J, H, K, Q)$ as follows:

- $(J, H)=\left(J_{1}, H_{1}\right) \&\left(J_{2}, H_{2}\right)$.

- $K=K_{1} \times K_{2}$

\footnotetext{
${ }^{5} B^{\prime}$ is "more general" than $B$.
} 
- $Q\left(k_{1}, k_{2}\right)=\left\{\vec{d}_{1} \times \vec{d}_{2} \mid \vec{d}_{1} \in Q_{1}\left(k_{1}\right), \vec{d}_{2} \in Q_{2}\left(K_{2}\right)\right\}$ where $\left(\vec{d}_{1} \times \vec{d}_{2}\right)\left(i n_{1}(j)\right)=\vec{d}_{1}(j),\left(\vec{d}_{1} \times \vec{d}_{2}\right)\left(i n_{2}(j)\right)=\vec{d}_{2}(j)$

Fact $4.9 B_{1} \& B_{2}$ is failure iff $B_{1}$ is failure or $B_{2}$ is failure.

Definition 4.10 Given specializations $s_{1}$ from $B_{1}$ to $B_{1}^{\prime}$ and $s_{2}$ from $B_{2}$ to $B_{2}^{\prime}$. Let $B_{1}=\left(J_{1}, H_{1}, K_{1}, Q_{1}\right), B_{2}=\left(J_{2}, H_{2}, K_{2}, Q_{2}\right), B_{1}^{\prime}=\left(J_{1}, H_{1}, k_{1}^{\prime}, Q_{1}^{\prime}\right)$ and $B_{2}^{\prime}=\left(J_{2}, H_{2}, K_{2}^{\prime}, Q_{2}^{\prime}\right)$. Then define $s=s_{1} \& s_{2}$, a specialization from $B_{1} \& B_{2}$ to $B_{1}^{\prime} \& B_{2}^{\prime}$, by

$$
\left(s_{1} \& s_{2}\right)\left(k_{1}, k_{2}\right)=\left\{\left(k_{1}^{\prime}, k_{2}^{\prime}\right) \mid k_{1}^{\prime} \in s_{1}\left(k_{1}\right), k_{2}^{\prime} \in s_{2}\left(k_{2}\right)\right\}
$$

We have to check that this actually is a specialization: but with $B_{1} \& B_{2}=$ $(J, H, K, Q)$ and $B_{1}^{\prime} \& B_{2}^{\prime}=\left(J, H, K^{\prime}, Q^{\prime}\right)$ we have

$$
\begin{aligned}
Q\left(k_{1}, k_{2}\right) & =\left\{\vec{d}_{1} \times \vec{d}_{2} \mid \vec{d}_{1} \in Q_{1}\left(k_{1}\right), \vec{d}_{2} \in Q_{2}\left(k_{2}\right)\right\} \\
& =\left\{\vec{d}_{1} \times \vec{d}_{2} \mid \exists k_{1}^{\prime} \in s_{1}\left(k_{1}\right), \exists k_{2}^{\prime} \in s_{2}\left(k_{2}\right): \vec{d}_{1} \in Q_{1}^{\prime}\left(k_{1}^{\prime}\right), \vec{d}_{2} \in Q_{2}^{\prime}\left(k_{2}^{\prime}\right)\right\} \\
& =\left\{\vec{d} \mid \exists k_{1}^{\prime} \in s_{1}\left(k_{1}\right), \exists k_{2}^{\prime} \in s_{2}\left(k_{2}\right): \vec{d} \in Q^{\prime}\left(k_{1}^{\prime}, k_{2}^{\prime}\right)\right\} \\
& =\left\{\vec{d} \mid \exists k^{\prime} \in s\left(k_{1}, k_{2}\right): \vec{d} \in Q^{\prime}\left(k^{\prime}\right)\right\} \\
& =\bigcup_{k^{\prime} \in s\left(k_{1}, k_{2}\right)} Q^{\prime}\left(k^{\prime}\right)
\end{aligned}
$$

Definition 4.11 Given specialization $s$ from $B$ to $B^{\prime}$, and specialization $s^{\prime}$ from $B^{\prime}$ to $B^{\prime \prime}$, we define $s \star s^{\prime}$, a specialization from $B$ to ", by (here $\left.B=(J, H, K, Q), B^{\prime}=\left(J, H, K^{\prime}, Q^{\prime}\right), B^{\prime \prime}=\left(J, H, K^{\prime \prime}, Q^{\prime \prime}\right)\right)$

$$
\left(s \star s^{\prime}\right)(k)=\bigcup_{k^{\prime} \in s(k)} s^{\prime}\left(k^{\prime}\right)
$$

We have to check that this actually is a specialization:

$$
Q(k)=\bigcup_{k^{\prime} \in s(k)} Q^{\prime}\left(k^{\prime}\right)=\bigcup_{k^{\prime} \in s(k)} \bigcup_{k^{\prime \prime} \in s^{\prime}\left(k^{\prime}\right)} Q^{\prime \prime}\left(k^{\prime \prime}\right)=\bigcup_{k^{\prime \prime} \in\left(s \star s^{\prime}\right)(k)} Q^{\prime \prime}\left(k^{\prime \prime}\right)
$$

Definition 4.12 Given basic configuration $B=(J, H, K, Q)$ we define $I d_{B}$, a specialization from $B$ to $B$, by

$$
I d_{B}(k)=\{k\}
$$




\section{Algebraic identities}

When writing "=", we always mean "module isomorphism". It should be obvious what it means for two basic configurations to be isomorphic.

Fact 4.13 By letting the objects be basic configurations and by letting the morphisms be specializations, we obtain a category. That is, $\star$ is associative and $I d_{B}$ is a neutral element for all $B$.

Moreover, $\&$ is a functor in this category - i.e. $I d_{B_{1}} \& I d_{B_{2}}=I d_{B_{1} \& B_{2}}$, and $\left(s_{1} \star s_{1}^{\prime}\right) \&\left(s_{2} \star s_{2}^{\prime}\right)=\left(s_{1} \& s_{2}\right) \star\left(s_{1}^{\prime} \& s_{2}^{\prime}\right)$.

Finally, \& is associative and $C a_{H_{1}} \& C a_{H_{2}}=C a_{H_{1} \& H_{2}}$.

PRoOF: The only nontrivial part is the relation between $\&$ and $\star$ :

$$
\begin{aligned}
& \left(k_{1}^{\prime \prime}, k_{2}^{\prime \prime}\right) \in\left(\left(s_{1} \star s_{1}^{\prime}\right) \&\left(s_{2} \star s_{2}^{\prime}\right)\right)\left(k_{1}, k_{2}\right) \\
\Leftrightarrow & k_{1}^{\prime \prime} \in\left(s_{1} \star s_{1}^{\prime}\right)\left(k_{1}\right), k_{2}^{\prime \prime} \in\left(s_{2} \star s_{2}^{\prime}\right)\left(k_{2}\right) \\
\Leftrightarrow & \exists k_{1}^{\prime}, k_{2}^{\prime}: k_{1}^{\prime} \in s_{1}\left(k_{1}\right), k_{1}^{\prime \prime} \in s_{1}^{\prime}\left(k_{1}^{\prime}\right), k_{2}^{\prime} \in s_{2}\left(k_{2}\right), k_{2}^{\prime \prime} \in s_{2}^{\prime}\left(k_{2}^{\prime}\right) \\
\Leftrightarrow & \exists\left(k_{1}^{\prime}, k_{2}^{\prime}\right):\left(k_{1}^{\prime}, k_{2}^{\prime}\right) \in\left(s_{1} \& s_{2}\right)\left(k_{1}, k_{2}\right),\left(k_{1}^{\prime \prime}, k_{2}^{\prime \prime}\right) \in\left(s_{1}^{\prime} \& s_{2}^{\prime}\right)\left(k_{1}^{\prime}, k_{2}^{\prime}\right) \\
\Leftrightarrow & \left(k_{1}^{\prime \prime}, k_{2}^{\prime \prime} \in\left(\left(s_{1} \& s_{2}\right) \star\left(s_{1}^{\prime} \& s_{2}^{\prime}\right)\right)\left(k_{1}, k_{2}\right)\right.
\end{aligned}
$$

\section{$4.2 \quad$ U-mirrors}

Given a function $O I$ which for each $G \in U$ returns a non-empty and finite index set $O I(G)$.

Given a function $A I$ which for each $G \in U$ and each $i \in O I(G)$ returns a finite index set $A I(G)$, equipped with a total order $<$.

Given a function $P$ which for each $G \in U, i \in O I(G)$ and $j \in A I(G, i)$ returns $P(G, i, j) \in U$.

Given a function $W$ which for each $G \in U, i \in O I(G)$ and $j \in A I(G, i)$ returns $W(G, i, j)$, a non-negative integer.

Returning to example 1.1, there e.g. $O I(f)=\{1,2\}$ (or any two-element set); $O I(g)=\{1\}$ (or any one-element set), $A I(f, 1)=\emptyset, A I(g)=\{1,2\}$ with $1<2, P(g, 1,1)=P(g, 1,2)=f$. 


\section{U-forests}

Definition 4.14 A $U$-forest from goal sequence $(J, H)$ to goal sequence $\left(J^{\prime}, H^{\prime}\right)$ is a $J$-indexed family of trees where

1. Nodes are labeled by a goal label $G, G \in U$. Some nodes are also equipped with an or-direction label $i$ with $i \in O I(G)$. All nodes not being leaves, and possibly also some leaves, have an or-direction label.

2. Arcs are labeled by an and-direction label $j$ and a weight label $w$. Distinct arcs going from the same node are labeled by distinct anddirection labels.

3. For all $j \in J$, the root of the $j$ 'th tree has goal label $H(j)$.

4. Let $N$ be a node which has an or-direction label $i$, and which has goal label $G$. Then $j$ will be the and-direction label of an arc going from $N$ iff $j \in A I(G, i)$. The arcs from $N$ inherit the ordering of $A I(G, i)$.

5. Let $a$ be an arc from a node $N$, with goal label $G$, to $N^{\prime}$. Then $G$ will contain an or-direction label $i$. Further, with $j$ the and-direction label and $w$ the weight label of $a$, the goal label of $N^{\prime}$ is $P(G, i, j)$ and $w=W(G, i, j)$.

6. There is a total ordering among the leaves - and thus also among the paths, where a path starts at a root and ends at a leaf - determined in the "natural way" by the ordering on $J$ and the ordering on the arcs leaving each node.

7. The sequence of leaves not having an or-direction label, together with their goal labels, is isomorphic to $\left(J^{\prime}, H^{\prime}\right)$.

- A path ending in a leaf not having an or-direction label is termed working.

- A U-forest is working iff all paths are working.

- The weight of a path $p, W(p)$, is the sum of the weight labels encountered when walking along $p$.

- The weight of a U-forest $f, W(f)$, is the sum of the weight labels in $f$. 
To be more formal, a working path $p$ in a $\mathrm{U}$-forest from $(J, H)$ to $\left(J^{\prime}, H^{\prime}\right)$ is a sequence of the form

$j G_{0}\left(i_{1}, j_{1}, w_{1}\right) G_{1} \cdots\left(i_{n}, j_{n}, w_{n}\right) j^{\prime} G_{n}(n \geq 0)$

where $j \in J, G_{0}=H(j), j^{\prime} \in J^{\prime}, G_{n}=H^{\prime}\left(j^{\prime}\right), G_{k}=P\left(G_{k-1}, i_{k}, j_{k}\right)$ and $w_{k}=W\left(G_{k-1} i_{k}, j_{k}\right)$ for $k=1 \ldots n$.

A non-working path in a U-forest from $(J, H)$ to $\left(J^{\prime}, H^{\prime}\right)$ is a sequence of the form

$j G_{0}\left(i_{1}, j_{1}, w_{1} G_{1} \cdots\left(i_{n}, j_{n}, w_{n}\right) G_{n} i(n \geq 0)\right.$

where $j \in J, G_{0}=H(j), A I\left(G_{n}, i\right)=\emptyset, G_{k}=P\left(G_{k-1}, i_{k}, j_{k}\right)$ and. $w_{k}=$ $W\left(G_{k-1}, i_{k}, j_{k}\right)$ for $k=1 \ldots n$.

In both cases $W(p)=\sum_{k=1}^{n} w_{k}$.

Definition 4.15 If $p$ is a working path in a U-forest from $(J, H)$ to $\left(J^{\prime}, H^{\prime}\right)$ of form $j G q j^{\prime} G^{\prime}$, and $p^{\prime}$ is a path in a U-forest from $\left(J^{\prime}, H^{\prime}\right)$ to $\left(J^{\prime \prime}, H^{\prime \prime}\right)$ of form $j^{\prime} G^{\prime} q^{\prime}$, then we define $p \star p^{\prime}=j G q j^{\prime} G^{\prime} q^{\prime}$.

Definition 4.16 Given U-forest $f$ from $(J, H)$ to $\left(J^{\prime}, H^{\prime}\right)$ and U-forest $f^{\prime}$ from $\left(J^{\prime}, H^{\prime}\right)$ to $\left(J^{\prime \prime}, H^{\prime \prime}\right)$. We can now define $f \star f^{\prime}$, a U-forest from $(J, H)$ to $\left(J^{\prime \prime}, H^{\prime \prime}\right)$, by "gluing" the two forests together in the obvious way.

Observation 4.17 Given a path $p^{\prime \prime}$ in $f \star f^{\prime}$. Two possibilities:

- $p^{\prime \prime}$ is a non-working path in $f$. Then $p^{\prime \prime}$ will be non-working in $f \star f^{\prime}$ as well.

- There exists working path $p$ in $f$ and path $p^{\prime}$ in $f^{\prime}$ such that $p^{\prime \prime}=p \star p^{\prime}$. $p^{\prime \prime}$ will be working iff $p^{\prime}$ is. These $p$ and $p^{\prime}$ are unique.

Conversely, if $p^{\prime}$ is a path in $f^{\prime}$ there exists exactly one (working) path $p$ in $f$ such that $p \star p^{\prime}$ forms a path in $f \star f^{\prime}$. If $p$ is a working path in $f$, there exists at least one path $p^{\prime}$ in $f^{\prime}$ such that $p \star p^{\prime}$ forms a path in $f \star f^{\prime}$.

Definition 4.18 Given goal sequence $(J, H) . I d_{(J, H)}$ is now defined as the U-forest from $(J, H)$ to $(J, H)$, where all paths ire of the form $j G$. 
Definition 4.19 Given U-forest $f_{1}$ from $H_{1}$ to $H_{1}^{\prime}$ and U-forest $f_{2}$ from $H_{2}$ to $H_{2}^{\prime}$. Now define $f_{1} \& f_{2}$, a U-forest from $H_{1} \& H_{2}$ to $H_{1}^{\prime} \& H_{2}^{\prime}$, in the obvious way - i.e. the paths in $f_{1} \& f_{2}$ will be the "disjoint union" of the paths in $f_{1}$ and the paths in $f_{2}$.

Fact 4.20 By letting the objects be goal sequences and the morphisms be U-forests, one gets a category (with $\star$ as composition and $I d_{-}$as identities). $\&$ is a functor in this category, and $\&$ is associative.

Definition 4.21 Given a U-forest $f_{1}$ from $H$ to $H_{1}$, and a U-forest $f_{2}$ from $H$ to $H_{2}$. We say that $\left(f_{1}^{\prime}, f_{2}^{\prime}, H^{\prime}\right)$ is a completion of $\left(f_{1}, f_{2}\right)$ if $f_{1}^{\prime}$ is a U-forest from $H_{1}$ to $H^{\prime}, f_{2}^{\prime}$ is a U-forest from $H_{2}$ to $H^{\prime}$, and $f_{1} \star f_{1}^{\prime}=f_{2} \star f_{2}^{\prime}$.

\section{Pushouts}

Observation 4.22 Given a U-forest $f_{1}$ from $H$ to $H_{1}$, and a U-forest $f_{2}$ from $H$ to $H_{2}$. Suppose $\left(f_{1}, f_{2}\right)$ has a completion. Then there exists a completion $\left(f_{1}^{\prime}, f_{2}^{\prime}, H^{\prime}\right)$ such that for all completions $\left(f_{1}^{\prime \prime}, f_{2}^{\prime \prime}, H^{\prime \prime}\right)$ there exists a U-forest $f$ from $H^{\prime}$ to $H^{\prime \prime}$ with $f_{1}^{\prime} \star f=f_{1}^{\prime \prime}, f_{2}^{\prime} \star f=f_{2}^{\prime \prime}$. Consequently, this completion is unique - we term $\left(f_{1}^{\prime}, f_{2}^{\prime}, H^{\prime}\right)$ the pushout of $\left(f_{1}, f_{2}\right)$.

Moreover, if $f_{1}$ is working then $f_{2}^{\prime}$ will be working.

Fact 4.23 Taking pushouts is commutative in the following sense: given $\left(f_{1}, f_{1}^{\prime}\right)$ and $\left(f_{2}, f_{2}^{\prime}\right)$, such that $f_{1}^{\prime}$ and $f_{2}$ are U-forests to the same goal sequence. Suppose $\left(f_{1}, f_{1}^{\prime}\right)$ has pushout $\left(f_{3}, f_{3}^{\prime}\right)$, and suppose $\left(f_{2} \star f_{3}^{\prime}, f_{2}^{\prime}\right)$ has pushout $\left(f_{4}, f_{4}^{\prime}\right)$. Now the situation is as in the left part of figure 4 .

Then $\left(f_{2}, f_{2}^{\prime}\right)$ will have a pushout, to be written $\left(f_{5}, f_{5}^{\prime}\right)$; and also $\left(f_{1}, f_{1}^{\prime} \star f_{5}\right)$ will have a pushout, to be written $\left(f_{6}, f_{6}^{\prime}\right)$ - as depicted in the right part of figure 4. Moreover,

$f_{3} \star f_{4}=f_{6}, f_{5}^{\prime} \star f_{6}^{\prime}=f_{4}^{\prime}$

Proof: Standard categorical reasoning is applied: As $f_{2} \star f_{3}^{\prime} \star f_{4}=f_{2}^{\prime} \star f_{4}^{\prime}$ we see that $\left(f_{2}, f_{2}^{\prime}\right)$ in fact has a pushout $\left(f_{5}, f_{5}^{\prime}\right)$, and there exists unique $f$ such that $f_{5} \star f=f_{3}^{\prime} \star f_{4}, f_{5}^{\prime} \star f=f_{4}^{\prime}$. Now

$f_{1} \star f_{3} \star f_{4}=f_{1}^{\prime} \star f_{3}^{\prime} \star f_{4}=f_{1}^{\prime} \star f_{5} \star f$ 

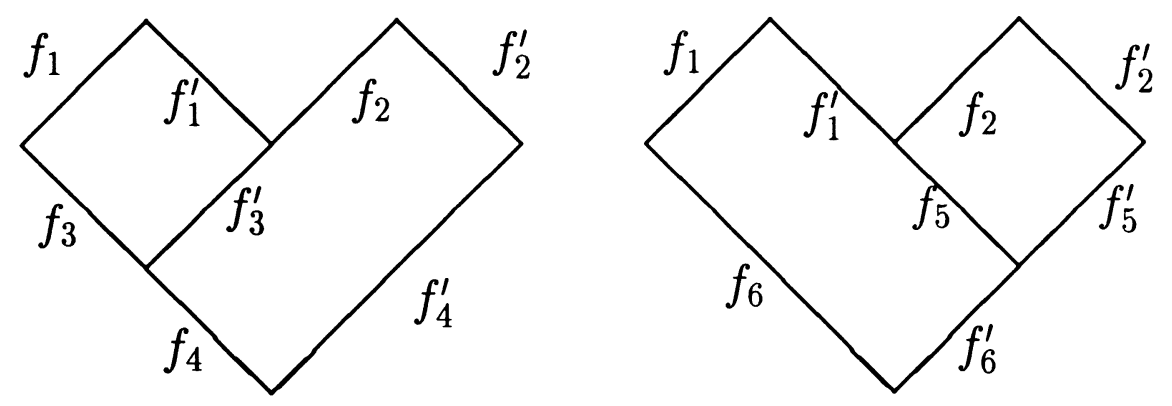

Figure 4: Pushout commutes

which shows that $\left(f_{1}, f_{1}^{\prime} \star f_{5}\right)$ has a pushout $\left(f_{6}, f_{6}^{\prime}\right)$, and there exists $f^{\prime}$ such that $f_{6} \star f^{\prime}=f_{3} \star f_{4}, f_{6}^{\prime} \star f^{\prime}=f$.

Also we have $f_{5}^{\prime} \star f_{6}^{\prime} \star f^{\prime}=f_{5}^{\prime} \star f=f_{4}^{\prime}$. For reasons of symmetry ( $f_{6}$ plays the same role as $f_{4}^{\prime}, f_{5}^{\prime}$ plays the same role as $f_{3}$ and $f_{6}^{\prime}$ plays the same role as $f_{4}$ ) we can find $f^{\prime \prime}$ such that $f_{4}^{\prime} \star f^{\prime \prime}=f_{5}^{\prime} \star f_{6}^{\prime} f_{3} \star f_{4} \star f^{\prime \prime}=f_{6}$.

From $f_{6}=f_{3} \star f_{4} \star f^{\prime \prime}=f_{6} \star f^{\prime} \star f^{\prime \prime}$ we conclude $f^{\prime}=I d_{-}$- hence the claim.

\section{U-mirrors}

Definition 4.24 A $U$-mirror $m$ from goal sequence $H$ to goal sequence $H^{\prime}$ is a triple $\left(f, f^{\prime}, H^{\prime \prime}\right)^{6}$ where $f$ is a U-forest from $H$ to $H^{\prime \prime}$; and where $f^{\prime}$ is a U-forest from $H^{\prime}$ to $H^{\prime \prime}$. We require $f^{\prime}$ to be working (as we do not allow folding using a rule of form $g \leftarrow \square$ ).

- One can in the natural way define the paths of a U-mirror. A path $p^{\prime \prime}$ is either a non-working path in $f$ or of the form $\left(p, p^{\prime}\right)$ where $p$ is a working path in $f$ of form $q j^{\prime \prime} G^{\prime \prime}$ and $p^{\prime}$ a (working) path in $f^{\prime}$ of form $q^{\prime} j^{\prime \prime} G^{\prime \prime}$ - we say $p$ and $p^{\prime}$ are connected. Paths of the former kind are termed non-working; paths of the latter kind are termed working.

- We say that $m$ is working iff all paths are working - i.e. iff $f$ is working.

- The weight of a non-working path $p, W(p)$, is the weight of $p$ in $f$; the

\footnotetext{
${ }^{6}$ We often just write $\left(f, f^{\prime}\right)$ and omit $H^{\prime \prime}$.
} 
weight of a working path $\left(p, p^{\prime}\right), W\left(p, p^{\prime}\right)$, is the difference between the weight of $p$ in $f$ and the weight of $p^{\prime}$ in $f^{\prime}$.

- The weight of the U-mirror, $W(m)$, is $W(f)-W\left(f^{\prime}\right)$.

Observe that given working path $p$ in $f$ there exists exactly one path $p^{\prime}$ in $f^{\prime}$ connected to $p$; and given (working) path $p^{\prime}$ in $f^{\prime}$ there exists exactly one path $p$ in $f$ connected to $p^{\prime}$. Also, there will exist a working path in $m$ iff $H^{\prime}$ is not empty.

We will not distinguish between a U-forest $f$ from $H$ to $H^{\prime}$ and the Umirror $\left(f, I d_{H^{\prime}}, H^{\prime}\right)$.

Definition 4.25 Let $m=\left(f, f^{\prime}, H^{\prime \prime}\right)$ be a U-mirror from $H$ to $H^{\prime}$, and suppose $m$ is working (i.e. $f$ is working). Then we can define $\mathcal{R}(m)$, a (working) U-mirror from $H^{\prime}$ to $H$, by $\mathcal{R}(m)=\left(f^{\prime}, f, H^{\prime \prime}\right)$.

Definition 4.26 Let $m_{1}=\left(f_{1}, f_{1}^{\prime}, H_{1}^{\prime \prime}\right)$ be a U-mirror from $H_{1}$ to $H_{1}^{\prime}$, and let $m_{2}=\left(f_{2}, f_{2}^{\prime}, H_{2}^{\prime \prime}\right)$ be a U-mirror from $H_{2}$ to $H_{2}^{\prime}$. Now we can define $m_{1} \& m_{2}$, a U-mirror from $H_{1} \& H_{2}$ to $H_{1}^{\prime} \& H_{2}^{\prime}$, by letting

$m_{1} \& m_{2}=\left(f_{1} \& f_{2}, f_{1}^{\prime} \& f_{2}^{\prime}, H_{1}^{\prime \prime} \& H_{2}^{\prime \prime}\right)$

Definition 4.27 Given goal sequence $H$. Then we can define $I d_{H}$ as $\left(I d_{H}\right.$, $\left.I d_{H}, H\right)$.

Definition 4.28 Given a U-mirror $m_{1}=\left(f_{11}, f_{21}, H_{1}^{\prime}\right)$ from $H_{1}$ to $H_{2}$, and given a U-mirror $m_{2}=\left(f_{22}, f_{32}, H_{2}^{\prime}\right)$ from $H_{2}$ to $H_{3}$.

First suppose the pushout of $\left(f_{21}, f_{22}\right)$ exists. Let it be $\left(f_{1}^{\prime}, f_{2}^{\prime}, H^{\prime \prime}\right)$. Then

$m_{1} \star m_{2}=\left(f_{11} \star f_{1}^{\prime}, f_{32} \star f_{2}^{\prime}, H^{\prime \prime}\right)$

(This is a well-defined U-mirror: $m_{1}$ is a U-mirror, so $f_{21}$ is working. By observation 4.22, $f_{2}^{\prime}$ is working. As $m_{2}$ is a U-mirror, $f_{32}$ is working. Hence, $f_{32} \star f_{2}^{\prime}$ is working.)

If the pushout does not exist, $m_{1} \star m_{2}=\perp$ (i.e. undefined).

Lemma 4.29 Suppose $m_{1} \star m_{2} \neq \perp$. Suppose $\left(p, p^{\prime}\right)$ is a path in $m_{1} \star m_{2}$. Then there exists paths $\left(p_{1}, p_{1}^{\prime}\right)$ in $m_{1},\left(p_{2}, p_{2}^{\prime}\right)$ in $m_{2}$ and paths $p^{\prime \prime}, p^{\prime \prime \prime}$ such that $p=p_{1} \star p^{\prime \prime}, p^{\prime}=p_{2}^{\prime} \star p^{\prime \prime \prime}$, and $p_{1}^{\prime} \star p^{\prime \prime}=p_{2} \star p^{\prime \prime \prime}$. 
Proof: Let $m_{1}=\left(f_{1}, f_{1}^{\prime}\left(J_{1}^{\prime}, H_{1}^{\prime}\right)\right)$ be a U-mirror from $\left(J_{1}, H_{1}\right)$ to $\left(J_{2}, H_{2}\right)$, and let $m_{2}=\left(f_{2}, f_{2}^{\prime}\left(J_{2}^{\prime}, H_{2}^{\prime}\right)\right)$ b e a U-mirror from $\left(J_{2}, H_{2}\right)$ to $\left(J_{3}, H_{3}\right)$. Let $\left(f^{\prime \prime}, f^{\prime \prime \prime},\left(J^{\prime \prime}, H^{\prime \prime}\right)\right)$ be the pushout of $\left(f_{1}^{\prime}, f_{2}\right)$. Then

$m_{1} \star m_{2}=\left(f_{1} \star f^{\prime \prime}, f_{2}^{\prime} \star f^{\prime \prime \prime}\right)$

We have that $p$ is a working path in $f_{1} \star f^{\prime \prime}$, and $p^{\prime}$ is a (working) path in $f_{2}^{\prime} \star f^{\prime \prime \prime}$. There thus exist working paths $p_{1}$ in $f_{1}, p^{\prime \prime}$ in $f^{\prime \prime}, p_{2}^{\prime}$ in $f_{2}^{\prime}$ and $p^{\prime \prime \prime}$ in $f^{\prime \prime \prime}$ such that $p=p_{1} \star p^{\prime \prime}, p^{\prime}=p_{2}^{\prime} \star p^{\prime \prime \prime}$. Now let $p_{2}$ in $f_{2}$ and $p_{1}^{\prime}$ in $f_{1}^{\prime}$ be the unique paths connected to $p_{2}^{\prime}$ and $p_{1}$ respectively. As there exists $j_{1}^{\prime} \in J_{1}^{\prime}$ such that $p_{1}$ and $p_{1}^{\prime}$ both end with $j_{1}^{\prime} H_{1}^{\prime}\left(j_{1}^{\prime}\right), p_{1}^{\prime} \star p^{\prime \prime}$ will be a path in $f_{1}^{\prime} \star f^{\prime \prime}$. Similarly, $p_{2} \star p^{\prime \prime \prime}$ will be a path in $f_{2} \star f^{\prime \prime \prime}$. Since $f_{1}^{\prime} \star f^{\prime \prime}=f_{2} \star f^{\prime \prime \prime}$, and since $p_{2} \star p^{\prime \prime \prime}$ ends with the same element in $J^{\prime \prime}$ as $p^{\prime \prime \prime}$ does as $p^{\prime}$ does as $p$ does as $p^{\prime \prime}$ does as $p_{1}^{\prime} \star p^{\prime \prime}$ does, we conclude that actually $p_{1}^{\prime} \star p^{\prime \prime}=p_{2} \star p^{\prime \prime \prime}$.

\section{Fact 4.30}

1. Operating on U-mirrors, $\star$ is associative ${ }^{7}$ with $I d_{-}$as neutral element.

2. \& is a functor; i.e. $\left(m_{1} \star m_{1}^{\prime}\right) \&\left(m_{2} \star m_{2}^{\prime}\right)=\left(m_{1} \& m_{2}\right) \star\left(m_{1}^{\prime} \& m_{2}^{\prime}\right)$; and $I d_{H} \& I d_{H^{\prime}}=I d_{H \& H^{\prime}}$. Also, \& is associative.

3. The property of being working is closed under all operations in question.

4. Given working $f$ from $H$ to $H^{\prime}$. Considered as a U-mirror, $\mathcal{R}(f) \star f=$ $I d_{H^{\prime}}$.

Proof: First for the last claim: we must show that $\left(I d_{H^{\prime}}, f\right) \star\left(f, I d_{H^{\prime}}\right)=$ $I d_{H^{\prime}}$. Now the pushout of $(f, f)$ is $\left(I d_{H^{\prime}}, I d_{H^{\prime}}\right)$, hence the claim follows.

That $I d_{-}$is neutral element can be seen as follows: Let $m=\left(f, f^{\prime}, H^{\prime \prime}\right)$ be a U-mirror from $H$ to $H^{\prime}$. The pushout of $\left(f^{\prime}, I d_{H^{\prime}}\right)$ clearly is $\left(I d_{H^{\prime \prime}}, f^{\prime}, H^{\prime \prime}\right)$. So

$m \star I d_{H^{\prime}}=\left(f \star I d_{H^{\prime \prime}}, I d_{H^{\prime}} \star f^{\prime}, H^{\prime \prime}\right)=\left(f, f^{\prime}, H^{\prime \prime}\right)=m$

That $I d_{-}$is left neutral element is seen similarly.

\footnotetext{
${ }^{7}$ Equality, in the presence of $\perp$, means that either both sides are $\perp$ or both sides are $\neq \perp$ and equal.
} 

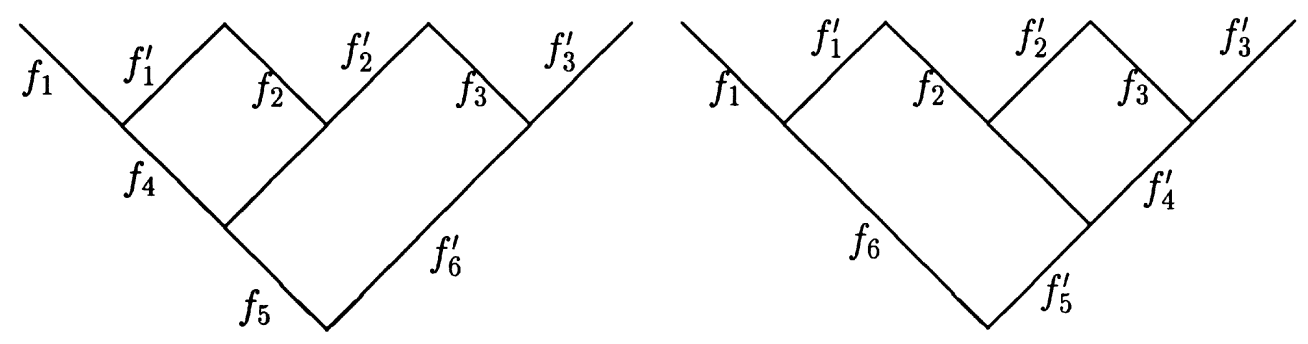

Figure 5: Associativity of $\star$ on U-mirrors

Next for the associativity: let $m_{1}=\left(f_{1}, f_{1}^{\prime}\right), m_{2}=\left(f_{2}, f_{2}^{\prime}\right), m_{3}=\left(f_{3}, f_{3}^{\prime}\right)$. Consider figure 5, where the relevant pushouts have been drawn (assuming they exist). Then

$\left(m_{1} \star m_{2}\right) \star m_{3}=\left(f_{1} \star f_{4} \star f_{5}, f_{3}^{\prime} \star f_{6}^{\prime}\right)$

$m_{1} \star\left(m_{2} \star m_{3}\right)=\left(f_{1} \star f_{6}, f_{3}^{\prime} \star f_{4}^{\prime} \star f_{5}^{\prime}\right)$

Now we can apply fact 4.23 to see that $\left(m_{1} \star m_{2}\right) \star m_{3}$ will be defined iff $m_{1} \star\left(m_{2} \star m_{3}\right)$ is, and then they will be equal.

The remaining claims are trivial.

\subsection{Properties of U-mirrors}

Definition 4.31 Given $n$, we say that a U-mirror $m$ satisfies $\mathcal{F}(n)$ iff for all working paths $p$ we have $W(p) \geq n$; and there exists a working path.

Lemma 4.32 If $m_{1}$ satisfies $\mathcal{F}\left(n_{1}\right)$ and $m_{2}$ satisfies $\mathcal{F}\left(n_{2}\right)$ then $m_{1} \star m_{2}$ - if defined - satisfies $\mathcal{F}\left(n_{1}+n_{2}\right)$.

Proof: Let $m_{1}=\left(f_{1}, f_{1}^{\prime}, H_{1}^{\prime}\right)$ from $H_{1}$ to $H_{2}$; and let $m_{2}=\left(f_{2}, f_{2}^{\prime}, H_{2}^{\prime}\right)$ from $\mathrm{H}_{2}$ to $\mathrm{H}_{3}$.

Since there exists a working path in $m_{2}, H_{3}$ is not empty, and hence there exists a working path in $m_{1} \star m_{2}$.

Let $\left(p, p^{\prime}\right)$ be a working path in $m_{1} \star m_{2}$. By lemma 4.29 , there exists $\left(p_{1}, p_{1}^{\prime}\right)$ in $m_{1},\left(p_{2}, p_{2}^{\prime}\right)$ in $m_{2}$ and paths $p^{\prime \prime}, p^{\prime \prime \prime}$ such that $p=p_{1} \star p^{\prime \prime}, p^{\prime}=p_{2}^{\prime} \star p^{\prime \prime \prime}$, and $p_{1}^{\prime} \star p^{\prime \prime}=p_{2} \star p^{\prime \prime \prime}$. Now, by applying the assumption, we have

$W\left(p, p^{\prime}\right)=W(p)-W\left(p^{\prime}\right)=W\left(p_{1}\right)+W\left(p^{\prime \prime}\right)-W\left(p_{2}^{\prime}\right)-W\left(p^{\prime \prime \prime}\right)$ 


$$
\begin{aligned}
& \geq W\left(p_{1}^{\prime}\right)+n_{1}+W\left(p^{\prime \prime}\right)-W\left(p_{2}\right)+n_{2}-W\left(p^{\prime \prime \prime}\right) \\
& =W\left(p_{1}^{\prime} \star p^{\prime \prime}\right)-W\left(p_{2} \star p^{\prime \prime \prime}\right)+n_{1}+n_{2} \\
& =n_{1}+n_{2}
\end{aligned}
$$

Definition 4.33 Given $n$, we say that a U-mirror $m$ satisfies $\mathcal{A}(n)$ iff $W(m)$ $\geq n$.

Lemma 4.34 If $m_{1}$ satisfies $\mathcal{A}\left(n_{1}\right)$, and $m_{2}$ satisfies $\mathcal{A}\left(n_{2}\right)$, then $m_{1} \star m_{2}$ if defined - satisfies $\left.\mathcal{A}\left(n_{1}\right)+m_{2}\right)$.

Proof: Let $m_{1}=\left(f_{1}, f_{1}^{\prime}, H_{1}^{\prime}\right)$ from $H_{1}$ to $H_{2}$; and let $m_{2}=\left(f_{2}, f_{2}^{\prime}, H_{2}^{\prime}\right)$ from $H_{2}$ to $H_{3}$. Let $\left(f^{\prime \prime}, f^{\prime \prime \prime}\right)$ be the pushout of $\left(f_{1}^{\prime}, f_{2}\right)$. Now

$$
\begin{aligned}
W\left(m_{1} \star m_{2}\right) & =W\left(f_{1} \star f^{\prime \prime}\right)-W\left(f_{2}^{\prime} \star f^{\prime \prime \prime}\right) \\
& =W\left(f_{1}\right)+W\left(f^{\prime \prime}\right)-W\left(f_{2}^{\prime}\right)-W\left(f^{\prime \prime \prime}\right) \\
& \geq W\left(f_{1}^{\prime}\right)+n_{1}+W\left(f^{\prime \prime}\right)-W\left(f_{2}\right)+n_{2}-W\left(f^{\prime \prime \prime}\right) \\
& =W\left(f_{1}^{\prime} \star f^{\prime \prime}\right)-W\left(f_{2} \star f^{\prime \prime \prime}\right)+n_{1}+n_{2} \\
& =n_{1}+n_{2}
\end{aligned}
$$

Definition 4.35 Given $n$, we say that a U-mirror $m$ satisfies $\mathcal{L}(n)$ iff there exists a path in $m$, and the leftmost path has weight $\geq n$.

Definition 4.36 Given a U-mirror $m$, we let

- $E(m)$ denote the number of non-working paths to the left, i.e. $E(m) \geq$ $n$ iff the $\mathrm{n}$ leftmost paths in $m$ are non-working.

- $L(m)$ denote the weight of the leftmost working path (if no such exists, $0)$.

Then we can define an ordering $\prec$ on the set of U-mirrors by letting $m_{1} \prec m_{2}$ iff $E\left(m_{1}\right)<E\left(m_{2}\right)$ or $E\left(m_{1}\right)=E\left(m_{2}\right)$ and $L\left(m_{1}\right)<L\left(m_{2}\right)$.

Lemma 4.37 Suppose $m_{2}$ satisfies $\mathcal{L}(1)$, and $m=m_{1} \star m_{2}$ is defined. Then $m_{1} \prec m$. 
PROOF: First some notation: we say that a path is left-directed if the following holds for all its arcs: let the arc have and-direction label $j$ and go from a node with goal label $G$ and or-direction label $i$. Then $j$ is the least element in $A I(G, i)$.

Let $m_{1}=\left(f_{1}, f_{1}^{\prime}\right), m_{2}=\left(f_{2}, f_{2}^{\prime}\right)$. Let $\left(f^{\prime \prime}, f^{\prime \prime \prime}\right)$ be the pushout of $\left(f_{1}^{\prime}, f_{2}\right)$. Now $m=\left(f_{1} \star f^{\prime \prime}, f_{2}^{\prime} \star f^{\prime \prime \prime}\right)$. The $E\left(m_{1}\right)$ leftmost paths of $m_{1}$ will be nonworking; so the $E\left(m_{1}\right)$ leftmost paths of $f_{1}$ will be non-working; so the $E\left(m_{1}\right)$ leftmost paths of $f_{1} \star f^{\prime \prime}$ will be non-working; so the $E\left(m_{1}\right)$ leftmost paths of $m$ will be non-working. This shows that $E\left(m_{1}\right) \leq E(m)$.

Now consider the leftmost path in $m_{2}$. Two possibilities:

- It is of form $p_{2}$ with $p_{2}$ non-working in $f_{2}$. As $f_{2} \star f^{\prime \prime \prime}=f_{1}^{\prime} \star f^{\prime \prime}$, there will exist $p_{1}^{\prime}$ in $f_{1}^{\prime}$ and left-directed $p^{\prime \prime}$ in $f^{\prime \prime}$ such that $p_{2}=p_{1}^{\prime} \star p^{\prime \prime}$, and such that $p_{1}^{\prime}$ is the leftmost path in $f^{\prime}-1$. Let $p_{1}^{\prime}$ be connected to $p_{1} ; p_{1}$ will be the leftmost working path in $f_{1}$. Now $p_{1} \star p^{\prime \prime}$ will be non-working in $f_{1} \star f^{\prime \prime}$ and hence also in $m$. This shows that $E\left(m_{1}\right)<E(m)$ and hence $m_{1} \prec m$.

- It is of form $\left(p_{2}, p_{2}^{\prime}\right)$ with $p_{2}$ working in $f_{2}$. As $f_{2} \star f^{\prime \prime \prime}=f_{1}^{\prime} \star f^{\prime \prime}$, there will exist $p_{1}^{\prime}$ in $f_{1}^{\prime}$, left-directed $p^{\prime \prime}$ in $f^{\prime \prime}$ and left-directed $p^{\prime \prime \prime}$ in $f^{\prime \prime \prime}$ such that $p_{2} \star p^{\prime \prime \prime}=p_{1}^{\prime} \star p^{\prime \prime}$, and such that $p_{1}^{\prime}$ is the leftmost path in $f_{1}^{\prime}$. Let $p_{1}^{\prime}$ be connected to $p_{1} ; p_{1}$ will be the leftmost working path in $f_{1}$. Now $\left(p_{1} \star p^{\prime \prime}, p_{2}^{\prime} \star p^{\prime \prime \prime}\right)$ will belong to $m$, and be the leftmost working path in $m$. Moreover,

$$
\begin{aligned}
L(m) & =W\left(p_{1} \star p^{\prime \prime}\right)-W\left(p_{2}^{\prime} \star p^{\prime \prime \prime}\right) \\
& =W\left(p_{1}\right)+W\left(p^{\prime \prime}\right)-W\left(p_{2}^{\prime}\right)-W\left(p^{\prime \prime \prime}\right) \\
& >W\left(p_{1}\right)+W\left(p^{\prime \prime}\right)-W\left(p_{2}\right)-W\left(p^{\prime \prime \prime}\right) \\
& =W\left(p_{1}\right)+W\left(p^{\prime \prime}\right)-W\left(p_{2} \star p^{\prime \prime \prime}\right) \\
& =W\left(p_{1}\right)+W\left(p^{\prime \prime}\right)-W\left(p_{1}^{\prime} \star p^{\prime \prime}\right) \\
& =W\left(p_{1}\right)-W\left(p_{1}^{\prime}\right) \\
& =L\left(m_{1}\right)
\end{aligned}
$$

This shows $m_{1} \prec m$. 


\subsection{Transitions}

Definition 4.38 A transition $t$ from basic configuration $B=(J, H, K, Q)$ to $B^{\prime}=\left(J^{\prime}, H^{\prime}, K^{\prime}, Q^{\prime}\right)$ (notice the $K$-sets are identical) is a set of U-mirrors from $(J, H)$ to $\left(J^{\prime}, H^{\prime}\right)$ which is either empty or a singleton. ${ }^{8}$ We will demand that $\left(Q, Q^{\prime}\right)$ is a non-increasing pair, i.e. that for all $k \in K, Q(k)=\emptyset \Rightarrow$ $Q^{\prime}(k)=\emptyset$ (so it will not be possible to make a transition from a failure basic configuration into a non-failure, cf. the discussion in section 2.4, (1)).

We say that $t$ is stable iff also $\left(Q^{\prime}, Q\right)$ is a non-increasing pair, i.e. $Q(k)=$ $\emptyset \Leftrightarrow Q^{\prime}(k)=\emptyset$.

Definition 4.39 Let $t$ be a transition from $B$ to $B^{\prime}$, and let $t^{\prime}$ be a transition from $B^{\prime}$ to $B^{\prime \prime}$. Now define $t \star t^{\prime}$, a transition from $B$ to $B^{\prime \prime}$, by

$m^{\prime \prime} \in t \star t^{\prime} \Leftrightarrow \exists m \in t, m^{\prime} \in t^{\prime}: m^{\prime \prime}=m \star m^{\prime}$

Clearly $\left(B, B^{\prime \prime}\right)$ is a non-increasing pair, and $t \star t^{\prime}$ will be stable if $t$ and $t^{\prime}$ are stable.

Definition 4.40 Let $t_{1}$ be a transition from $B_{1}$ to $B_{1}^{\prime}$, and let $t_{2}$ be a transition from $B_{2}$ to $B_{2}^{\prime}$. Now define $t_{1} \& t_{2}$, a transition from $B_{1} \& B_{2}$ to $B_{1}^{\prime} \& B_{2}^{\prime}$, by

$m \in t_{1} \& t_{2} \Leftrightarrow \exists m_{1} \in t_{1}, m_{2} \in t_{2}: m=m_{1} \& m_{2}$

Clearly non-increasingness will be preserved, and $t_{1} \& t_{2}$ will be stable if $t_{1}$ and $t_{2}$ are stable.

Definition 4.41 Given $B=(J, H, K, Q)$, define $I d_{B}$, a transition from $B$ to $B$, by letting $I d_{B}$ be the singleton set containing $I d_{J, H}$. This is clearly stable.

Definition 4.42 Given a transition $t$ from $B$ to $B^{\prime}$. Let $s$ be a specialization from $\mathcal{I}_{s}(B)$ to $B$. Now we define $\mathcal{I}_{s}(t)$, a transition from $\mathcal{I}_{s}(B)$ to $\mathcal{I}_{s}\left(B^{\prime}\right)$, by letting $m$ be in $\mathcal{I}_{s}(t)$ iff $m$ is in $t$. Clearly $\left(\mathcal{I}_{s}(B), \mathcal{I}_{s}\left(B^{\prime}\right)\right)$ is a non-increasing pair, and $\mathcal{I}_{s}(t)$ will be stable if $t$ is.

Definition 4.43 Given a transition $t$ from $B$ to $B^{\prime}$. Suppose $t$ is stable, and suppose it for all $m \in t$ holds that $m$ is working. Then we say that $t$ is

\footnotetext{
${ }^{8}$ We will often identify $t$ with its element.
} 
reversible and we can define $\mathcal{R}(t)$, a transition from $B^{\prime}$ to $B$, by stipulating that $m$ is in $\mathcal{R}(t)$ iff $\mathcal{R}(m)$ is in $t$. Clearly $\mathcal{R}(t)$ is reversible.

Fact 4.44 By letting the objects be basic configurations and by letting the morphisms be transitions, we obtain a category. \& is a functor in this category.

\section{Two level transition system}

\subsection{The level 0 rules}

Assume that for all $G \in U$ and $i \in O I(G)$ there exists a transition $t(G, i)$ from $C a_{G}$ to $c(G, i)$, where $c(G, i)$ takes the form $(A I(G, i), \lambda j . P(G, i, j), \mathcal{D})$

and where $t(G, i)$ contains the U-mirror (U-forest) determined by the paths

$\left\{G\left(i, j_{,-}\right) G^{\prime} \mid j \in A I(G, i)\right\}$

where $G^{\prime}=P(G, i, j)$. However, if $A I(G, i)=\emptyset$ the U-mirror will be determined by the singleton path Gi. Clearly, this in non-increasing. Now

$$
\mathcal{R} \mathcal{U}_{0}=\{t(G, i) \mid G \in U, i \in O I(G)\}
$$

In example 1.1, e.g. $c(g, 1)=([f, f j], \mathcal{D} \times \mathcal{D}, Q)$ with

$Q\left(d_{1}, d_{2}\right)=\left\{\left\{\left(\left(d_{1}, d\right),\left(d, d_{2}\right)\right)\right\} \mid d \in \mathcal{D}\right\}$

\subsection{Unfolding at level 1}

We now define what it means for a transition $t$ from $B$ to $B^{\prime}$ to be a level 1 unfolding step, to be written $1 \vdash_{u}^{S} t: B \rightarrow B^{\prime 9}$ (here $H_{1}$ and $H_{2}$ are goal sequences, and $s$ is a specialization):

\footnotetext{
${ }^{9}$ When we are not interested in the configurations, we may simply write $1 \vdash{ }_{u}^{S} t$.
} 
$\frac{t(G, i) \in \mathcal{R} \mathcal{U}_{0}}{1 \vdash_{u}^{S} \mathcal{I}_{s}\left(I d C_{H_{1}} \& t(G, i) \& I d_{C a_{H_{2}}}\right)}$

Next we define what it means for a transition $t$ from $B$ to $B^{\prime}$ to be a level 1 unfolding, to be written $1 \vdash_{u} T: B \rightarrow B^{\prime}$

$$
\begin{aligned}
& \frac{1 \vdash_{u}^{S} t: B \rightarrow B^{\prime}}{1 \vdash_{u} t: B \rightarrow B^{\prime}} \\
& \frac{1 \vdash_{u} I d_{B}: B \rightarrow B}{1 \vdash_{u} t: B \rightarrow B^{\prime}, 1 \vdash_{u} t^{\prime}: B^{\prime} \rightarrow B^{\prime \prime}} \\
& \frac{1 \vdash_{u} t \star t^{\prime}: B \rightarrow B^{\prime \prime}}{}
\end{aligned}
$$

If $1 \vdash_{u} t$, we can write $t=t_{1} \star \cdots t_{n}(n \geq 0)$ with $1 \vdash_{u}^{S} t_{i}$ for $i \in\{1 \ldots n\}$. The least $n$ which can be used is called the length of $t$.

\section{Fact 5.1}

1. If $1 \vdash_{u}^{S} t$, then $1 \vdash_{u}^{S} t \& I d_{B}$ and $1 \vdash_{u}^{S} I d_{B} \& t$ for basic configuration $B$.

2. If $1 \vdash_{u}^{S} t$, then $1 \vdash_{u}^{S} \mathcal{I}_{s}(t)$ for specialization $s$.

3. If $1 \vdash_{u} t$, then $1 \vdash_{u} t \& I d_{B}$ and $1 \vdash_{u} I d_{B} \& t$ for basic configuration $B$.

4. If $1 \vdash_{u} t_{1}$ and $1 \vdash_{u} t_{2}$, then $1 \vdash_{u} t_{1} \& t_{2}$.

5. If $1 \vdash_{u} t$, then $1 \vdash_{u} \mathcal{I}_{s}(t)$ for specialization $s$.

6. If $1 \vdash_{u} t$, then $t$ is a singleton - i.e. of form $\{m\}$.

PRoOF: For (1), notice that fact 4.5 tells us that we can write $B=\mathcal{I}_{s^{\prime}}\left(C a_{H}\right)$. Then

$$
\begin{aligned}
& \mathcal{I}_{s}\left(I d_{C a_{H_{1}}} \& t(G, i) \& I d_{C a_{H_{2}}}\right) \& I d_{B} \\
= & \mathcal{I}_{s}\left(I d_{C a_{H_{1}}} \& t(G, i) \& I d_{C a_{H_{2}}}\right) \& \mathcal{I}_{s^{\prime}}\left(I d C a_{H}\right) \\
= & \mathcal{I}_{s \& s^{\prime}}\left(I_{C a_{H_{1}}} \& t(G, i) \& I d_{C a_{H_{2}}} \& I d C a_{H}\right) \\
= & \mathcal{I}_{s \& s^{\prime}}\left(I d_{C a_{H_{1}}} \& t(G, i) \& I d_{C a_{H_{2} \& H}}\right)
\end{aligned}
$$

(2) is trivial. (3) follows by induction in the derivation tree for $1 \vdash_{u} t$ : the case where rule (5) has b een applied follows from what has just been 
shown; the case where rule (6) has been applied is trivial; and the case where rule (7) has been applied follows from the calculation (cf. fact 4.44)

$\left(t \& I d_{B}\right) \star\left(t^{\prime} \& I d_{B}\right)=\left(t \star t^{\prime}\right) \&\left(I d_{B} \star I d_{B}\right)=\left(t \star t^{\prime}\right) \& I d_{B}$

(4) follows from what has been just shown and fact 4.44:

$t_{1} \& t_{2}=\left(t_{1} \star I d_{-}\right) \&\left(I d_{-} \star t_{2}\right)=\left(t_{1} \& I d_{-}\right) \star\left(I d_{-} \& t_{2}\right)$

(5) is a trivial induction in the derivation tree. (6) follows from the fact that all U-mirrors involved in fact are U-forests, thus * can never be undefined.

\section{The diamond lemma}

Lemma 5.2 Suppose $1 \vdash_{u}^{S} t_{1}: B \rightarrow B_{1}$ and $1 \vdash_{u}^{S} t_{2}: B \rightarrow B_{2}$. Suppose $t_{1}$ and $t_{2}$ have a completion (viewed as U-forests). Then there exists $B^{\prime}$, transition $t_{1}^{\prime}$ from $B_{1}$ to $B_{1}^{\prime}$ and transition $t_{2}$ from $B_{2}$ to $B_{2}^{\prime}$ such that $\left(t_{1}^{\prime}, t_{2}^{\prime}\right)$ (viewed as a U-forest) is the pushout of $\left(t_{1}, t_{2}\right)$. Moreover, one of two holds:

- $B_{1}=B_{2}=B^{\prime}, t_{1}=t_{2}, t_{1}^{\prime}=t_{2}^{\prime}=I d_{B^{\prime}}$.

- $1 \vdash_{u}^{S} t_{1}^{\prime}: B_{1} \rightarrow B^{\prime}$ and $1 \vdash_{u}^{S} t_{2}^{\prime}: B_{2} \rightarrow B^{\prime}$.

Proof: We can assume that the transitions involved are of the following form:

$$
\begin{aligned}
& t_{1}=\mathcal{I}_{s_{1}}\left(I d_{C a_{H_{11}}} \& t\left(G_{1}, i_{1}\right) \& I d_{C a_{H_{12}}}\right) \\
& t_{2}=\mathcal{I}_{s_{2}}\left(I_{C a_{H_{21}}} \& t\left(G_{2}, i_{2}\right) \& I d_{C a_{H_{22}}}\right)
\end{aligned}
$$

As $t_{1}$ and $t_{2}$ both are transitions from $B$, we get

$\mathcal{I}_{s_{1}}\left(C a_{H_{11}} \& G_{1} \& H_{12}\right)=B=\mathcal{I}_{s_{2}}\left(C a_{H_{21}} \& G_{2} \& H_{22}\right)$

From this we infer $H_{11} \& G_{1} \& H_{12}=H_{21} \& G_{2} \& H_{22}$ and (by fact 45 ) that $s_{1}=s_{2}$. Now two possibilities: 
- $H_{11}=H_{21}$. Then $G_{1}=G_{2}$, and $H_{12}=H_{22}$. As $\left(t_{1}, t_{2}\right)$ has a completion, $i_{1}=i_{2}$. This shows $t_{1}=t_{2}$ and $B_{1}=B_{2}$. Thus we can choose $t_{1}^{\prime}=t_{2}^{\prime}=I d_{B_{1}}$, and clearly $\left(t_{1}^{\prime}, t_{2}^{\prime}\right)$ is the pushout.

- $H_{11} \neq H_{21}$. We can wlog. assume that $H_{11}$ is shorter than $H_{21}$. Then there exists $H$ such that $H_{21}=H \& G_{2} \& H_{22}, H_{21}=H_{11} \& G_{1} \& H$. Now define

$$
\begin{aligned}
& t_{1}^{\prime}=\mathcal{I}_{s_{1}}\left(I_{C a_{H_{11}}} \& I d_{c\left(G_{1}, i_{1}\right)} \& I d_{C a_{H}} \& t\left(G_{2}, i_{2}\right) \& I d C a_{H_{22}}\right) \\
& t_{2}=\mathcal{I}_{s_{1}}\left(I_{C a_{H_{11}}} \& t\left(G_{1}, i_{1}\right) \& I d_{C a_{H}} \& I_{c\left(G_{2}, i_{2}\right.}, \& I d a_{H_{22}}\right)
\end{aligned}
$$

We can easily calculate

$$
\begin{aligned}
t_{1} \star t_{1}^{\prime} & =\mathcal{I}_{s_{1}}\left(I d d_{C a_{H_{11}}} \& t\left(G_{1}, i_{1}\right) \& I d_{C a_{H}} \& t\left(G_{2}, i_{2}\right) \& I d{ }_{C a_{H_{22}}}\right. \\
& =t_{2} \star t_{2}^{\prime}
\end{aligned}
$$

To show that $1 \vdash_{u}^{S} t_{1}^{\prime}$, notice that we can write $c\left(G_{1}, i_{1}\right)$ on the form $\mathcal{I}_{s^{\prime}}\left(C a_{H^{\prime}}\right)$ for some $s^{\prime}, H^{\prime}$ - similarly we have $1 \vdash_{u}^{S} t_{2}^{\prime}$

Again, viewed as U-forests $\left(t_{1}^{\prime}, t_{2}^{\prime}\right)$ is clearly the pushout.

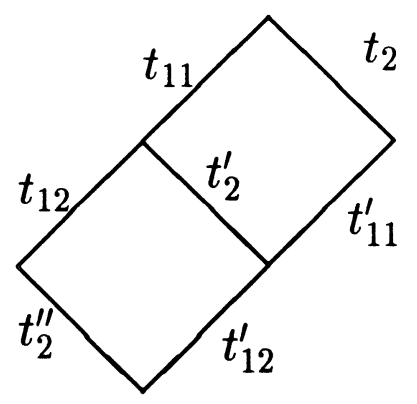

Figure 6: The Church-Rosser property

\section{The Church-Rosser lemma}

Lemma 5.3 Suppose $1 \vdash_{u} t_{1}: B \rightarrow B_{1}$ and $1 \vdash_{u} t_{2}: B \rightarrow B_{2}$. Suppose $t_{1}$ and $t_{2}$ have a completion, viewed as U-forests. Then there exists $B^{\prime}$ and transitions $t_{1}^{\prime}, t_{2}^{\prime}$ such that $1 \vdash_{u} t_{1}^{\prime}: B_{1} \rightarrow B^{\prime}, 1 \vdash_{u} t_{2}^{\prime}: B_{2} \rightarrow B^{\prime}$, and such that $\left(t_{1}^{\prime}, t_{2}^{\prime}\right)$ - viewed as U-forests - is the pushout of $\left(t_{1}, t_{2}\right)$. 
Moreover, the length of $t_{1}^{\prime}$ is less than or equal the length of $t_{2}$, and the length of $t_{2}^{\prime}$ is less than or equal the length of $t_{1}$.

PROOF: Induction in the length of $t_{1}$ plus the lenght of $t_{2}$. If $t_{1}$ or $t_{2}$ is the identity the claim is clear. If $1 \vdash_{u}^{S} t_{1}$ and $1 \vdash_{u}^{S} t_{2}$, the claim follows from lemma 5.2.

Otherwise, we can wlog. assume that $t_{1}=t_{11} \star t_{12}$ with $1 \vdash_{u} t_{11}, 1 \vdash_{u} t_{12}$. The situation is as depicted in figure 6. By induction, there exists $t_{2}^{\prime}, t_{11}^{\prime}$ such that $1 \vdash_{u} t_{11}^{\prime}, 1 \vdash_{u} t_{2}^{\prime}$ and such that $\left(t_{2}^{\prime}, t_{11}^{\prime}\right)$ is the pushout of $\left(t_{11}, t_{2}\right)$. Moreover, the length of $t_{2}^{\prime}$ is less than or equal the length of $t_{2}$ - this enables us to use the induction hypothesis once more and arrive at $t_{2}^{\prime \prime}, t_{12}^{\prime}$ such that $1 \vdash_{u} t_{12}^{\prime}, 1 \vdash_{u} t_{2}^{\prime \prime}$.

Now we can choose $\left(t_{2}^{\prime \prime}, t_{11}^{\prime} \star t_{12}^{\prime}\right)$ as the desired transition pair. That it actually is the pushout of $\left(t_{1}, t_{2}\right)$ follows from fact 4.23 .

Lemma 5.3 shows that given a basic configuration $B=(J, H, K, Q)$ and an U-forest $f$ from $(J, H)$, it makes sense to define $\mathcal{U}_{f}(B)$ as the basic configuration $B^{\prime}$ such that $1 \vdash_{u} f: B \rightarrow B^{\prime}$.

\subsection{Evaluation strategies and Looping at level 1}

Definition 5.4 We say that $B$ loops at level 1 by some strategy if for all $n \geq 1$ third exists a $t_{n}$ and a $B_{n}$ not failure such that $1 \vdash_{u}^{S} t_{n}: B_{n-1} \rightarrow B_{n}$ (here $B_{0}=B$ ).

Lemma 5.5 The following are sufficient conditions for $B$ to loop at level 1 by some strategy:

1. For all $N \geq 0$, there for all $n$ with $1 \leq n \leq N$ exists $t_{n}$ and $B_{n}$ not failure such that $1 \vdash_{u}^{S} t_{n}: B_{n-1} \rightarrow B_{n}\left(\right.$ agin, $\left.B_{0}=B\right)$.

2. For all $n \geq 0$, there exists $B_{n}$ not failure and $t_{n}$ such that $1 \vdash_{u} t_{n}$ : $B \rightarrow B_{n}$, where $t_{n}$ viewed as a $\mathrm{U}$-forest has $n$ nodes with an or-direction label.

3. For all $n \geq 0$, there exists $B_{n}$ not failure and $t_{n}$ such that $1 \vdash_{u} t_{n}$ : $B \rightarrow B_{n}$, where $t_{n}$ viewed as a U-forest has weight $\geq n$ - i.e. satisfies $\mathcal{A}(n)$.

Proof: That (1) implies that $B$ loops at level 1 by some strategy is a consequence of Königs lemma (as there from a given $B$ is a finite number of 
level 1 unfolding steps, since $O I(G)$ is finite). That (2) implies (1) is obvious. That (3) implies (2) is a consequence of the weights being upper bounded (as $U, O I(G)$ and $A I(G, i)$ are finite sets).

\section{Fair strategy}

Definition 5.6 We say that $t$ is a fair level 1 unfolding step if $t$ is of form $\mathcal{I}_{s}\left(r_{1} \& \ldots \& r_{k}\right), k \geq 1$, where $r_{1} \ldots r_{k}$ are level 0 rules.

For a fair level 1 unfolding step $t$, we clearly have $1 \vdash_{u} t$.

Definition 5.7 We say that $B$ loops at level 1 by a fair strategy if for all $n \geq 1$ there exists a fair level 1 unfolding step $t_{n}$ and a $B_{n}$ not failure such $1 \vdash_{u} t_{n}: B_{n-1} \rightarrow B_{n}$ (here $B_{0}=B$ ).

Similar to lemma 5.5, we may prove

Lemma 5.8 The following are sufficient conditions for $B$ to loop at level 1 by a fair strategy:

1. For all $N \geq 0$, there for all $n$ with $1 \leq n \leq N$ exists a fair level 1 unfolding step $t_{n}$ and $B_{n}$ not failure such that $1 \vdash_{u} t_{n}: B_{n-1} \rightarrow B_{n}$ (again, $B_{0}=B$ ).

2. For all $n \geq 0$, there exists $B_{n}$ not failure and $t_{n}$ such that $1 \vdash_{u} t_{n}$ : $B \rightarrow B_{n}$, where each working path in $t_{n}$ (viewed as a U-forest) has length $\geq n$ and there exists a working path.

3. For all $n \geq 0$, there exists $B_{n}$ not failure and $t_{n}$ such that $1 \vdash_{u} t_{n}$ : $B \rightarrow B_{n}$, where $t_{n}$ satisfies $\mathcal{F}(n)$.

\section{$\mathcal{L} \mathcal{R}$ strategy}

Definition 5.9 We say that $t$ is a $\mathcal{L} \mathcal{R}$ level 1 unfolding step if $t$ is of form $\mathcal{I}_{s}\left(r \& I d_{-}\right)$with $r$ a level 0 rule.

We say that $t$ is a $\mathcal{L} \mathcal{R}$ level 1 unfolding if $t$ is of form $t_{1} \star \ldots \star t_{n}$ with each $t_{i}$ being a $\mathcal{L} \mathcal{R}$ level 1 unfolding step.

The U-forest corresponding to a $\mathcal{L} \mathcal{R}$ level 1 unfolding is termed a $\mathcal{L} \mathcal{R} U$ forest. 
Definition 5.10 We say that $B$ loops at level 1 by a $\mathcal{L} \mathcal{R}$ strategy if for all $n \geq 1$ there exists a $\mathcal{L} \mathcal{R}$ level 1 unfolding step $t_{n}$ and a $B_{n}$ not failure such that $1 \vdash_{u} t_{n}: B_{n-1} \rightarrow B_{n}$ (here $B_{0}=B$ ).

Recall the functions $E(m)$ and $L(m)$ introduced in section 4.3.

Lemma 5.11 The following are sufficient conditions for $B$ to loop at level 1 by a $\mathcal{L} \mathcal{R}$ strategy:

1. For all $N \geq 0$, there for all $n$ with $1 \leq n \leq N$ exists a $\mathcal{L} \mathcal{R}$ level 1 unfolding step $t_{n}$ and $B_{n}$ not failure such that $1 \vdash_{u}^{S} t_{n}: B_{n-1} \rightarrow B_{n}$ (again, $B_{0}=B$ ).

2. For all $n \geq 0$, there exists a transition $t_{n}$ and $B_{n}$ not failure, with $1 \vdash_{u} t_{n}: B \rightarrow B_{n}$, such that $E\left(t_{n}\right) \geq n$.

3. For all $n \geq 0$, there exists a transition $t_{n}$ and $B_{n}$ not failure, with $1 \vdash_{u} t_{n}: B \rightarrow B_{n}$, such that the length of the leftmost working path $\geq n$.

4. For all $n \geq 0$, there exists a transition $t_{n}$ and $B_{n}$ not failure, with $1 \vdash_{u} t_{n}: B \rightarrow B_{n}$, such that $L\left(t_{n}\right) \geq n$.

Proof: First consider (1); then $(2) \Rightarrow(1)$; then $(3) \Rightarrow(1)$ and finally (4) $\Rightarrow(3)$.

\subsection{Folding at level 1}

We now define what it means for a transition $t$ from $B$ to $B^{\prime}$ to be a level 1 folding step, to be written $1 \vdash_{f}^{S} t: B \rightarrow B^{\prime}$.

We will assume the existence of a partial function $s(G, i)$ such that $\mathcal{I}_{s(G, i)}$ $(t(G, i))$ is reversible, and such that $\mathcal{I}_{s(G, i)}\left(c\left(G, i^{\prime}\right)\right)$ is failure for all $i^{\prime} \neq i$.

$$
\frac{t(G, i) \in \mathcal{R} \mathcal{U}_{0}, s(G, i) \text { defined }}{1 \vdash_{f}^{S} \mathcal{I}_{s}\left(I_{C a_{H_{1}}} \& \mathcal{R}\left(\mathcal{I}_{s(G, i)}(t(G, i))\right) \& I d_{C a_{H_{2}}}\right)}
$$

Next we define what it means for a transition $t$ from $B$ to $B^{\prime}$ to be a level 1 folding, to be written $1 \vdash_{f} t: B \rightarrow B^{\prime}$ : 


$$
\begin{aligned}
& \frac{1 \vdash_{f}^{S} t: B \rightarrow B^{\prime}}{1 \vdash_{f} t: B \rightarrow B^{\prime}} \\
& \frac{1 \vdash_{f} I d_{B}: B \rightarrow B}{\frac{1 \vdash_{f} t: B \rightarrow B^{\prime}, 1 \vdash_{f} t^{\prime}: B^{\prime} \rightarrow B^{\prime \prime}}{1 \vdash_{f} t \star t^{\prime}: B \rightarrow B^{\prime \prime}}}
\end{aligned}
$$

\section{Fact 5.12}

1. If $1 \vdash_{f}^{S} t$, then $1 \vdash_{f}^{S} t \& I d_{B}$ and $1 \vdash_{f}^{S} I d_{B} \& t$ for basic configuration $B$.

2. If $1 \vdash_{f} t_{1}$ and $1 \vdash_{f} t_{2}$, then $1 \vdash_{f} t_{1} \& t_{2}$.

3. If $1 \vdash_{f}^{S} t\left(1 \vdash_{f} t\right)$, then $1 \vdash_{f}^{S} \mathcal{I}_{s}(t)\left(1 \vdash_{f} \mathcal{I}_{s}(t)\right)$ for specialization $s$.

4. If $1 \vdash_{f}^{S} t: B \rightarrow B^{\prime}\left(\right.$ or $\left.1 \vdash_{f} t: B \rightarrow B^{\prime}\right)$, then $t$ is reversible.

5. If $1 \vdash_{f}^{S} t: B \rightarrow B^{\prime}$ then $1 \vdash_{u}^{S} \mathcal{R}(t): B^{\prime} \rightarrow B$. If $\left.1 \vdash_{f} t: B \rightarrow B^{\prime}\right)$ then $1 \vdash_{u} \mathcal{R}(t): B^{\prime} \rightarrow B$.

ProOF: Mostly as in the proof of fact 5.1. The last point follows from

$$
\begin{aligned}
& \mathcal{R}\left(\mathcal{I}_{s}\left(I_{C a_{H_{1}}} \& \mathcal{R}\left(\mathcal{I}_{s(G, i)}(t(G, i))\right) \& I d_{C a_{H_{2}}}\right)\right) \\
= & \mathcal{I}_{s}\left(I d_{C a_{H_{1}}} \& \mathcal{I}_{s(G, i)}(t(G, i)) \& I d_{C a_{H_{2}}}\right) \\
= & \mathcal{I}_{s}\left(\mathcal{I}_{I d_{-}}\left(I_{C a_{H_{1}}}\right) \& \mathcal{I}_{s(G, i)}\left(t(G, i) \& \mathcal{I}_{I d_{-}}\left(I d_{C a_{H_{2}}}\right)\right)\right. \\
= & \mathcal{I}_{s}\left(\mathcal{I}_{I d_{-} \&_{s(G, i)} \& I d_{-}}\left({ }_{C d_{H_{1}}} \& t(G, i) \& I d_{C a_{H_{2}}}\right)\right) \\
= & \mathcal{I}_{s \star\left(I d_{-} \&_{s(G, i)} \& I d_{-}\right)}\left({ }^{\left(I d_{C a_{H_{1}}}\right.} \& t(G, i) \& I d_{C a_{H_{2}}}\right)
\end{aligned}
$$

\subsection{Unfold/fold at level 1}

We now define what it means for a transition $t$ from $B$ to $B^{\prime}$ to be a level 1 transition, to be written $1 \vdash t: B \rightarrow B^{\prime}$.

$$
\begin{aligned}
& \frac{\frac{1 \vdash_{u}^{S} B \rightarrow B^{\prime}}{1 \vdash t: B \rightarrow B^{\prime}}}{1 \vdash_{f}^{S} t: B \rightarrow B^{\prime}} \\
& 1 \vdash^{\prime} t: B \rightarrow B^{\prime}
\end{aligned}
$$




$$
\begin{aligned}
& \overline{1 \vdash I d_{B}: B \rightarrow B^{\prime}} \\
& \frac{1 \vdash t: B \rightarrow B^{\prime}, 1 \vdash t^{\prime}: B^{\prime} \rightarrow B^{\prime \prime}}{1 \vdash t \star t^{\prime}: B \rightarrow B^{\prime \prime}}
\end{aligned}
$$

\section{Fact 5.13}

1. If $1 \vdash t_{1}$ and $1 \vdash t_{2}$, then $1 \vdash t_{1} \& t_{2}$.

2. If $1 \vdash t$, then $1 \vdash \mathcal{I}_{s}(t)$ for specialization $s$.

\subsection{Fundamental properties of level 1 transitions}

\section{The switching lemma}

Lemma 5.14 Suppose $1 \vdash_{f}^{S} t_{1}: B_{1} \rightarrow B, 1 \vdash_{u}^{S} t_{2}: B \rightarrow B_{2}$, with $B_{2}$ not failure. Then one of two holds:

- $B_{1}=B_{2}, t_{1} \star t_{2}=I d_{B_{1}}$

- There exists $B^{\prime}, t_{1}^{\prime}, t_{2}^{\prime}$ with $1 \vdash_{u}^{S} t_{1}^{\prime}: B_{1} \rightarrow B^{\prime}, 1 \vdash_{f}^{S} t_{2}^{\prime}: B^{\prime} \rightarrow B_{2}$ such that

$$
t_{1} \star t_{2}=t_{1}^{\prime} \star t_{2}^{\prime}
$$

Proof: This lemma, as well as its proof, is very similar to lemma 5.2.

We can assume that the transitions involved are of the following form:

$$
\begin{aligned}
& t_{1}=\mathcal{I}_{s_{1}}\left(I d_{C a_{H_{11}}} \& \mathcal{R}\left(\mathcal{I}_{s\left(G_{1}, i_{1}\right)}\left(t\left(G_{1}, i_{1}\right)\right)\right) \& I d_{C a_{H_{12}}}\right) \\
& t_{2}=\quad \mathcal{I}_{s_{2}}\left(I_{C a_{H_{21}}} \& t\left(G_{2}, i_{2}\right) \& I d C_{H_{H_{22}}}\right)
\end{aligned}
$$

With $s=s_{1} \star\left(I d_{-} \& s\left(G_{1}, i_{1}\right) \& I d_{-}\right)$, we from the fact that $t_{1}$ is a transition to $B$ and $t_{2}$ is a transition from $B$ get

$\mathcal{I}_{s}\left(C a_{H_{11} \& G_{1} \& H_{12}}\right)=B=\mathcal{I}_{s_{2}}\left(C a_{H_{21} \& G_{2} \& H_{22}}\right)$

From this we infer $H_{11} \& G_{1} \& H_{12}=H_{21} \& G_{2} \& H_{22}$ and that $s=s_{2}$. Now two posse bilities:

- $H_{11}=H_{21}$. Then $G_{1}=G_{2}$, and $H_{12}=H_{22}$. Again two possibilities: 
$-i_{1} \neq i_{2}$. Then we have

$B_{2}=\mathcal{I}_{s_{1}}\left(C a_{H_{21}} \& \mathcal{I}_{s\left(G_{1}, i_{1}\right)}\left(c\left(G_{1}, i_{2}\right)\right) \& C a_{H_{22}}\right)$ but $\mathcal{I}_{s\left(G_{1}, i_{1}\right)}\left(c\left(G_{1}, i_{2}\right)\right)$ is failure by the definition of $s(G, i)$, hence $B_{2}$ is failure contradicting our assumption.

$-i_{1}=i_{2}$. Then it is obvious that $B_{1}=B_{2}$. And

$$
\begin{array}{rrr}
t_{1} \star t_{2} & = & \mathcal{I}_{s}\left(I d_{C a_{H_{11}}} \&\left(\mathcal{R}\left(t\left(G_{1}, i_{1}\right)\right) \star t\left(G_{1}, i_{1}\right)\right) \& I d_{C a_{H_{12}}}\right) \\
& = & \mathcal{I}_{s}\left(I d_{C a_{H_{11}}} \& I d_{c\left(G_{1}, i_{1}\right)} \& I d_{C a_{H_{12}}}\right) \\
= & I d_{\mathcal{I}_{s}\left(C a_{H_{11}} \& c\left(G_{1}, i_{1}\right) \& C a_{H_{12}}\right)}=I d_{B_{1}}
\end{array}
$$

where we have used fact $4.30,(4)$.

- $H_{11} \neq H_{21}$. We can wlog. assume that $H_{11}$ is shorter than $H_{21}$. Then there exists $H$ such that $H_{12}=H \& G_{2} \& H_{22}, H_{21}=H_{11} \& G_{1} \& H$. Now define

$$
\begin{aligned}
& t_{1}^{\prime}=\mathcal{I}_{s}\left(I_{C a_{H_{11}}} \& I d_{c\left(G_{1}, i_{1}\right.} \& I d_{C a_{H}} \& t\left(G_{2}, i_{2}\right) \& I d_{C a_{H_{22}}}\right) \\
& t_{2}^{\prime}=\mathcal{I}_{s_{1}}\left(I_{C a_{H_{11}}} \& \mathcal{R}\left(\mathcal{I}_{s\left(G_{1}, i_{1}\right)}\left(t\left(G_{1}, i_{1}\right)\right)\right) \& I d_{C a_{H}} \& \operatorname{Id}_{c\left(G_{2}, i_{2}\right)} \& I d_{C a_{H_{22}}}\right)
\end{aligned}
$$

We can easily calculate

$$
\begin{aligned}
& t_{1}^{\prime} \star t_{2}^{\prime} \\
= & \mathcal{I}_{s_{1}}\left(I d d_{H_{11}} \& \mathcal{R}\left(\mathcal{I}_{s\left(G_{1}, i_{1}\right)}\left(t\left(G_{1}, i_{1}\right)\right)\right) \& I d_{C a_{H}} \& t\left(G_{1}, i_{1}\right) \& I d_{C a_{H_{22}}}\right) \\
= & t_{1} \star t_{2}
\end{aligned}
$$

To show that $1 \vdash_{u}^{S} t_{1}^{\prime}$, notice that we can write $c\left(G_{1}, i_{1}\right)$ on the form $\mathcal{I}_{s^{\prime}}\left(C a_{H^{\prime}}\right)$ for some $s^{\prime}, H^{\prime}$. To show that $1 \vdash_{f}^{S} t_{2}^{\prime}$, apply the same observation to $c\left(G_{2}, i_{2}\right)$.

\section{The normalization lemma}

Lemma 5.15 Suppose $1 \vdash t: B \rightarrow B^{\prime}$, with $B^{\prime}$ not failure. Then there exists $B^{\prime \prime}, t_{1}$ and $t_{2}$ such that $1 \vdash_{u} t_{1}: B \rightarrow B^{\prime \prime}, 1 \vdash_{f} t_{2}: B^{\prime \prime} \rightarrow B^{\prime}, t_{1} \star t_{2}=t$.

From transitions being non-increasing we conclude that $B^{\prime \prime}$ is not failure, and from $t_{1}, t_{2}$ and $t_{1} \star t_{2}$ trivially being $\neq \perp$ we conclude $t \neq \perp$.

Proof: There exists $t_{1}, \ldots, t_{n}$ such that $t=t_{1} \star \cdots \star t_{n}$ and such that for each $i \in\{1 \ldots n\}$ either $1 \vdash_{u}^{S} t_{i}$ or $1 \vdash_{f}^{S} t_{i}$. We will use induction in 
the number of times an application of the fold-rule precedes (not necessarily immediately) an application of the unfold-rule. If this number is zero, we are through. Otherwise there exists $i, 1 \leq i \leq n$, such that $1 \vdash_{f}^{S} t_{i}, 1 \vdash_{u}^{S} t_{i+1}$. Now apply lemma 5.14. Two possibilities:

- $t_{i} \star t_{i+1}=I d_{-}$. Then

$t=t_{1} \star \cdots \star t_{i-1} \star t_{i+2} \star \cdots \star t_{n}$

with a strictly smaller number of "inversions".

- There exists $t_{i}^{\prime}, t_{i+1}^{\prime}$ such that $t_{i}^{\prime} \star t_{i+1}^{\prime}=t_{i} \star t_{i+1}$, and such that $1 \vdash_{u}^{S} t_{i}^{\prime}$, $1 \vdash_{u}^{S} t_{i+1}^{\prime}$. Now

$t=t_{1} \star \cdots \star t_{i-1} \star t_{i}^{\prime} \star t_{i+1}^{\prime} \star t_{i+2} \star \cdots \star t_{n}$

and again the number of inversions has decreased.

\subsection{Unfolding at level 2}

Now assume that we have defined $\mathcal{R} \mathcal{U}_{1}$, a finite set of rules at level 1 , such that $t \in \mathcal{R U}_{1}$ implies that $1 \vdash t$.

We now define what it means for a transition $t$ from $B$ to $B^{\prime}$ to be a level 2 unfolding step, to be written $2 \vdash^{S} t: B \rightarrow B^{\prime}$ :

$$
\frac{t \in \mathcal{R} \mathcal{U}_{1}}{2 \vdash^{S} \mathcal{I}_{s}\left(I d d_{C a_{1}} \& t \& I d C_{H_{H_{2}}}\right)}
$$

Next we define what it means for a transition $t$ from $B$ to $B^{\prime}$ to be a level 2 unfolding, to be written $2 \vdash t: B \rightarrow B^{\prime}$ :

$$
\begin{aligned}
& \frac{2 \vdash^{S} t: B \rightarrow B^{\prime}}{2 \vdash t: B \rightarrow B^{\prime}} \\
& \overline{2 \vdash I d_{B}: B \rightarrow B^{\prime}} \\
& \frac{2 \vdash t: B \rightarrow B^{\prime}, 2 \vdash t^{\prime}: B^{\prime} \rightarrow B^{\prime \prime}}{2 \vdash t \star t^{\prime}: B \rightarrow B^{\prime \prime}}
\end{aligned}
$$

Fact 5.16 Suppose $2 \vdash^{S} t$ or $2 \vdash t$. Then $1 \vdash t$. 
Proof: For $2 \vdash^{S} t$, exploit e.g. fact 5.13. For $2 \vdash t$, a straight forward induction in the derivation tree.

Definition 5.17 We say that $B$ loops at level 2 by some strategy if for all $n \geq 1$ there exists a $t_{n}$ and a $B_{n}$ not failure such that $2 \vdash^{S} t_{n}: B_{n-1} \rightarrow B_{n}$ (here $B_{0}=B$ ).

Definition 5.18 We say that $t$ is a fair level 2 step if $t$ is of form $\mathcal{I}_{s}\left(r_{1} \& \cdots \& r_{k}\right)$, $k \geq 1$, where $r_{1} \ldots r_{k}$ are level 1 rules.

For a fair level 2 step $t$, we clearly have $2 \vdash t$.

Definition 5.19 We say that $B$ loops at level 2 by a fair strategy if for all $n \geq 1$ there exists a fair level 2 step $t_{n}$ and a $B_{n}$ not failure such that $2 \vdash t_{n}: B_{n-1} \rightarrow B_{n}$ (here $B_{0}=B$ ).

Definition 5.20 We say that $t$ is a $\mathcal{L} \mathcal{R}$ level 2 step if $t$ is of form $\mathcal{I}_{s}\left(r \& I d_{-}\right)$ with $r$ a level 1 rule.

Definition 5.21 We say that $B$ loops at level 2 by a $\mathcal{L} \mathcal{R}$ strategy if for all $n \geq 1$ there exists a $\mathcal{L} \mathcal{R}$ level 2 step $t_{n}$ and a $B_{n}$ not failure such that $2 \vdash^{S} t_{n}: B_{n-1} \rightarrow B_{n}$ (here $B_{0}=B$ ).

\section{Conditions for termination preservation}

Theorem 6.1 Assume all rules in $\mathcal{R} \mathcal{U}_{1}$ satisfies $\mathcal{A}(1)$. Then if $B$ loops at level 2 by some strategy, it also loops at level 1 by some strategy.

Proof: Let, for all $n \geq 1$, be given $t_{n}$ and $B_{n}$ not failure such that $2 \vdash^{S} t_{n}: B_{n-1} \rightarrow B_{n}$. Define $t_{n}^{\prime}=t_{1} \star \cdots \star t_{n}$. Now $2 \vdash t_{n}^{\prime}: B \rightarrow B_{n}$, and by fact 5.16 also $1 \vdash t_{n}^{\prime}: B \rightarrow B_{n}$. By lemma 5.15 , there exists $t_{n}^{\prime \prime}, t_{n}^{\prime \prime \prime}$ and $B_{n}^{\prime}$ such that $1 \vdash_{u} t_{n}^{\prime \prime \prime}: B \rightarrow B_{n}^{\prime}, 1 \vdash_{f} t_{n}^{\prime \prime \prime}: B_{n}^{\prime} \rightarrow B_{n}, t_{n}^{\prime}=t_{n}^{\prime \prime} \star t_{n}^{\prime \prime \prime}$ and $B_{n}^{\prime}$ not failure.

Due to the assumption of the theorem, each $t_{i}$ will satisfy $\mathcal{A}(1)$. Then, by lemma 4.34 , each $t_{n}^{\prime}$ will satisfy $\mathcal{A}(n)$. But then also $t_{n}^{\prime \prime}$ will satisfy $\mathcal{A}(n)$. By lemma 5.5 , this shows that $B$ loops at level 1 by some strategy. 
Theorem 6.2 Assume all rules in $\mathcal{R} \mathcal{U}_{1}$ satisfies $\mathcal{F}(1)$. Then if $B$ loops at level 2 by a fair strategy, it also loops at level 1 by a fair strategy.

Proof: Let, for all $n \geq 1$, be given fair level 2 step $t_{n}$ and $B_{n}$ not failure such that $2 \vdash^{S} t_{n}: B_{n-1} \rightarrow B_{n}$. Define $t_{n}^{\prime}=t_{1} \star \cdots \star t_{n}$. Now $2 \vdash t_{n}^{\prime}: B \rightarrow B_{n}$, and by fact 5.16 also $1 \vdash t_{n}^{\prime}: B \rightarrow B_{n}$. By lemma 5.15. there exists $t_{n}^{\prime \prime}, t_{n}^{\prime \prime \prime}$ and $B_{n}^{\prime}$ such that $1 \vdash_{u} t_{n}^{\prime \prime}: B \rightarrow B_{n}^{\prime}, 1 \vdash_{f} t_{n}^{\prime \prime \prime}: B^{\prime}-n \rightarrow B_{n}, t_{n}^{\prime}=t_{n}^{\prime \prime} \star t_{n}^{\prime \prime \prime}$ and $B_{n}^{\prime}$ not failure.

Due to the assumption of the theorem, each $t_{i}$ will satisfy $\mathcal{F}(1)$. Then, by lemma 4.32, each $t_{n}^{\prime}$ will satisfy $\mathcal{F}(n)$. But then also $t_{n}^{\prime \prime}$ will satisfy $\mathcal{F}(n)$. By lemma 5.8, this shows that $B$ loops at level 1 by a fair strategy.

Theorem 6.3 Assume all rules in $\mathcal{R} \mathcal{U}_{1}$ satisfies $\mathcal{L}(1)$. Then if $B$ loops at level 2 by a $\mathcal{L R}$ strategy, it also loops at level 1 by a $\mathcal{L R}$ strategy.

Proof: Let, for all $n \geq 1$, be given $\mathcal{L R}$ level 2 step $t_{n}$ and $B_{n}$ not failure such that $2 \vdash^{S} t_{n}: B_{n-1} \rightarrow B_{n}$. Define $t_{n}^{\prime}=t_{1} \star \cdots \star t_{n}$. Now $2 \vdash t_{n}^{\prime}: B \rightarrow B_{n}$, and by fact 5.16 also $1 \vdash t_{n}^{\prime} B \rightarrow B_{n}$. By lemma 5.15 , there exists $t_{n}^{\prime \prime}, t_{n}^{\prime \prime \prime}$ and $B_{n}^{\prime}$ such that $1 \vdash_{u} t_{n}^{\prime \prime}: B \rightarrow B_{n}^{\prime}, 1 \vdash_{f} t_{n}^{\prime \prime \prime}: B_{n}^{\prime} \rightarrow B_{n}, t_{n}^{\prime}=t_{n}^{\prime \prime} \star t_{n}^{\prime \prime \prime}$ and $B_{n}^{\prime}$ not failure.

Due to the assumption of the theorem, each $t_{i}$ will satisfy $\mathcal{L}(1)$. Then, by lemma 4.37, we will have an increasing sequence

$t_{1}^{\prime} \prec t_{2}^{\prime} \prec t_{3}^{\prime} \prec \cdots$

Now two possibilities:

- $E\left(t_{n}^{\prime}\right)$ is not bounded. Then neither $E\left(t_{n}^{\prime \prime}\right)$ is bounded, so by lemma $5.11 B$ loops at level 1 by a $\mathcal{L R}$ strategy.

- $E\left(t_{n}^{\prime}\right)$ is bounded. Then $L\left(t_{n}^{\prime}\right)$ is unbounded, so also $L\left(t_{n}^{\prime \prime}\right)$ is unbounded. Then again lemma 5.11 tells us that $B$ loops at level 1 by a $\mathcal{L R}$ strategy. 


\section{Working with the full search tree}

Definition 7.1 A configuration $C$ (over $K$ ) is a family of basic configurations over $K$, i.e. consists of an index set $I$ and a mapping $B$ which to each $i \in I$ assigns a basic configuration over $K$.

Two new operators will be defined, + and $\mathrm{P}\left({ }_{-}\right)$:

Definition 7.2 Given configurations $C_{1}=\left(I_{1}, B_{1}\right)$ and $C_{2}=\left(I_{2}, B_{2}\right)$, over the same $K$. Now we define $C_{1}+C_{2}=(I, B)$ by letting $I=I_{1}+I_{2}$ (where + denotes disjoint union); and by letting $B\left(i n_{1}(i)\right)=B_{1}(i), B\left(i n_{2}(i)\right)=B_{2}(i)$.

Definition 7.3 Given configuration $C=(I, B)$. Let

$I^{\prime}=\{i \in I \mid B(i)$ is not failure $\}$

Now we define $\mathrm{P}(C)=\left(I^{\prime}, B^{\prime}\right)$ where $B^{\prime}\left(i^{\prime}\right)=B\left(i^{\prime}\right)$. We say that $C$ is pruned if $C=\mathrm{P}(C)$.

$\&$ will be extended to configurations such that \& distributes over + . That is, if $C=(I, B)$ and $C^{\prime}=\left(I^{\prime}, B^{\prime}\right)$ then $C \& C^{\prime}=\left(I \times I^{\prime}, B^{\prime \prime}\right)$ where $B^{\prime \prime}\left(i, i^{\prime}\right)=$ $B(i) \& B^{\prime}\left(i^{\prime}\right)$. Notice that this is possible only because configurations are multisets with + as multiset union; if configurations had been sequences with + as concatenation it would be impossible to make \& left-distributive as well as right-distributive.

$\mathcal{I}_{-}(-)$will be extended to configurations such that

$\mathcal{I}_{s}\left(C_{1}+C_{2}\right)=\mathcal{I}_{s}\left(C_{1}\right)+\mathcal{I}_{s}\left(C_{2}\right)$

A lot of new identities hold, not to be stated explicitly here - most are very trivial.

\subsection{Transitions}

Definition 7.4 A transition $t$ from $C=(I, B)$ to $C^{\prime}=\left(I^{\prime}, B^{\prime}\right)$ now to each $\left(i, i^{\prime}\right) \in I \times I^{\prime}$ assigns a set $t\left(i, i^{\prime}\right)$ of $\mathrm{U}$-mirrors from $B(i)$ to $B^{\prime}\left(i^{\prime}\right)$. Moreover, we will demand $t$ to be non-increasing: if $t\left(i, i^{\prime}\right)$ is non-empty, $\left(B(i), B^{\prime}\left(i^{\prime}\right)\right)$ must be a non-increasing pair. 
Definition 7.5 Given transition $t$ from $C=(I, B)$ to $C^{\prime}=\left(I^{\prime}, B^{\prime}\right)$ and transition $t^{\prime}$ from $C^{\prime}$ to $C^{\prime \prime}=\left(I^{\prime \prime}, B^{\prime \prime}\right) . t \star t^{\prime}$, a transition from $C$ to $C^{\prime \prime}$, is now defined as follows:

$\left(t \star t^{\prime}\right)\left(i, i^{\prime \prime}\right)=\left\{m \star m^{\prime} \mid \exists i^{\prime} \in I^{\prime}: m \in t\left(i, i^{\prime}\right), m^{\prime} \in t\left(i^{\prime}, i^{\prime \prime}\right)\right\}$

\& on transitions is defined in a similar vein:

Definition 7.6 Given transition $t_{1}$ from $C_{1}=\left(I_{1}, B_{1}\right)$ to $C_{1}^{\prime}=\left(I_{1}^{\prime}, B_{1}^{\prime}\right)$, and given transition $t_{2}$ from $C_{2}=\left(I_{2}, B_{2}\right)$ to $C_{2}^{\prime}=\left(I_{2}^{\prime}, B_{2}^{\prime}\right)$. Then $t_{1} \& t_{2}$, a transition from $C_{1} \& C_{2}$ to $C_{1}^{\prime} \& C_{2}^{\prime}$, is defined by

$\left(t_{1} \& t_{2}\right)\left(\left(i_{1}, i_{2}\right),\left(i_{1}^{\prime}, i_{2}^{\prime}\right)\right)=\left\{m_{1} \& m_{2} \mid m_{1} \in t_{1}\left(i_{1}, i_{1}^{\prime}\right), m_{2} \in t_{2}\left(i_{2}, i_{2}^{\prime}\right)\right\}$

Definition 7.7 Given transition $t$ from $C=(I, B)$ to $C^{\prime}=\left(I^{\prime}, B^{\prime}\right)$ Then $\mathcal{I}_{s}(t)$, a transition from $\mathcal{I}_{s}(C)$ to $\mathcal{I}_{s}\left(C^{\prime}\right)$, is given by

$\mathcal{I}_{s}(t)\left(i, i^{\prime}\right)=t\left(i, i^{\prime}\right)$

Definition 7.8 Given configuration $C=(I, B)$, we define $I d_{C}$ by

$I d_{C}(i, i)=\left\{I d_{B(i)}\right\}, I d_{C}\left(i, i^{\prime}\right)=\emptyset$ for $i \neq i^{\prime}$

Definition 7.9 Given transition $t_{1}$ from $C_{1}=\left(I_{1}, B_{1}\right)$ to $C_{1}^{\prime}=\left(I_{1}^{\prime}, B_{1}^{\prime}\right)$, and given transition $t_{2}$ from $C_{2}=\left(I_{2}, B_{2}\right)$ to $C_{2}^{\prime}=\left(I_{2}^{\prime}, B_{2}^{\prime}\right)$ (suppose $C_{1}$ and $C_{2}$ configurations over the same $K$ ). Then $t_{1}+t_{2}$, a transition from $C_{1}+C_{2}$ to $C_{1}^{\prime}+C_{2}^{\prime}$, is defined by

$$
\begin{aligned}
& \left(t_{1}+t_{2}\right)\left(i n_{1}\left(i_{1}, i n_{1}\left(i_{1}^{\prime}\right)\right)=t_{1}\left(i_{1}, i_{1}^{\prime}\right)\right. \\
& \left(t_{1}+t_{2}\right)\left(i n_{2}\left(i_{2}, i n_{2}\left(i_{2}^{\prime}\right)\right)=t_{2}\left(i_{2}, i_{2}^{\prime}\right)\right. \\
& \left(t_{1}+t_{2}\right)\left(i n_{1}\left(i_{1}, i n_{2}\left(i_{2}^{\prime}\right)\right)=\emptyset\right. \\
& \left(t_{1}+t_{2}\right)\left(i n_{2}\left(i_{2}, \quad i n_{1}\left(i_{1}^{\prime}\right)\right)=\emptyset\right.
\end{aligned}
$$

Definition 7.10 Given transition $t$ from $C_{1}=\left(I_{1}, B_{1}\right)$ to $C_{2}=\left(I_{2}, B_{2}\right)$. Let $\mathrm{P}\left(C_{1}\right)=\left(I_{1}^{\prime}, B_{1}^{\prime}\right), \mathrm{P}\left(C_{2}\right)=\left(I_{2}^{\prime}, B_{2}^{\prime}\right)$. Now define $\mathrm{P}(t)$, a transition from $\mathrm{P}\left(C_{1}\right)$ to $\mathrm{P}\left(C_{2}\right)$ by

$\mathrm{P}(t)\left(i_{1}^{\prime}, i_{2}^{\prime}\right)=t\left(i_{1}^{\prime}, i_{2}^{\prime}\right)$ 
Definition 7.11 Given transition $t$ from $C=(I, B)$ to $C^{\prime}=\left(I^{\prime}, B^{\prime}\right)$, we say that $t$ is reversible iff the following holds for all $\left(i, i^{\prime}\right)$ with $t\left(i, i^{\prime}\right) \neq \emptyset$ : $\left(B^{\prime}\left(i^{\prime}\right), B(i)\right)$ is a non-increasing pair, and for all $m \in t\left(i, i^{\prime}\right) m$ is a working U-mirror.

If $t$ is reversible we can define $\mathcal{R}(t)$, a transition from $C^{\prime}$ to $C$, by stipulating

$\mathcal{R}(t)\left(i^{\prime}, i\right)=\left\{\mathcal{R}(m) \mid m \in t\left(i, i^{\prime}\right)\right\}$

Again, a lot of algebraic identities hold - most are quite trivial. Let us just show that

$$
\left(t_{1} \& t_{2}\right) \star\left(t_{1}^{\prime} \& t_{2}^{\prime}\right)=\left(t_{1} \star t_{1}^{\prime}\right) \&\left(t_{2} \star t_{2}^{\prime}\right)
$$

where $t_{1}$ is a transition from $C_{1}=\left(I_{1}, B_{1}\right)$ to $C_{1}^{\prime}=\left(I_{1}^{\prime}, B_{1}^{\prime}\right)$, $t_{1}^{\prime}$ is a transition from $C_{1}^{\prime}$ to $C_{1}^{\prime \prime}=\left(I_{1}^{\prime \prime}, B_{1}^{\prime \prime}\right), t_{2}$ is a transition from $C_{2}=\left(I_{2}, B_{2}\right)$ to $C_{2}^{\prime}=\left(I_{2}^{\prime}, B_{2}^{\prime}\right)$ and $t_{2}^{\prime}$ is a transition from $C_{2}^{\prime}$ to $C_{2}^{\prime \prime}=\left(I_{2}^{\prime \prime}, B_{2}^{\prime \prime}\right)$. Now the left hand side of (20) as well as the right hand side will be a transition from $C_{1} \& C_{2}$ to $C_{1}^{\prime \prime} \& C_{2}^{\prime \prime}$. And for $i_{1} \in I_{1}, i_{2} \in I_{2}, i_{1}^{\prime \prime} \in I_{1}^{\prime \prime}$ and $i_{2}^{\prime \prime} \in I_{2}^{\prime \prime}$ we have

$$
\begin{aligned}
& \left(\left(t_{1} \& t_{2}\right) \star\left(t_{1}^{\prime} \& t_{2}^{\prime}\right)\right)\left(\left(i_{1}, i_{2}\right),\left(i_{1}^{\prime \prime}, i_{2}^{\prime \prime}\right)\right) \\
= & \left\{m^{\prime \prime} \mid \exists\left(i_{1}^{\prime}, i_{2}^{\prime}\right), \exists m \in\left(t_{1} \& t_{2}\right)\left(\left(i_{1}, i_{2}\right),\left(i_{1}^{\prime}, i_{2}^{\prime}\right)\right),\right. \\
& \left.\exists m^{\prime} \in\left(t_{1}^{\prime} \& t_{2}^{\prime}\right)\left(\left(i_{1}^{\prime}, i_{2}^{\prime}\right),\left(i_{1}^{\prime \prime}, i_{2}^{\prime \prime}\right)\right): m^{\prime \prime}=m \star m^{\prime}\right\} \\
= & \left\{m^{\prime \prime} \mid \exists\left(i_{1}^{\prime}, i_{2}^{\prime}\right), \exists m_{1} \in t_{1}\left(i_{1}, i_{1}^{\prime}\right), \exists m_{2} \in t_{2}\left(i_{2}, i_{2}^{\prime}\right),\right. \\
& \left.\exists m_{1}^{\prime} \in t_{1}^{\prime}\left(i_{1}^{\prime}, i_{1}^{\prime \prime}\right), \exists m_{2}^{\prime} \in t_{2}^{\prime}\left(i_{2}^{\prime}, i_{2}^{\prime \prime}\right): m^{\prime \prime}=\left(m_{1} \& m_{2}\right) \star\left(m_{1}^{\prime} \& m_{2}^{\prime}\right)\right\} \\
= & \left\{m^{\prime \prime} \mid \exists\left(i_{1}^{\prime}, i_{2}^{\prime}\right), \exists m_{1} \in t_{1}\left(i_{1}, i_{1}^{\prime}\right), e x i s t s m_{2} \in t_{2}\left(i_{2}, i_{2}^{\prime}\right),\right. \\
& \left.\exists m_{1}^{\prime} \in t_{1}^{\prime}\left(i_{1}^{\prime}, i_{1}^{\prime \prime}\right), \exists m_{2}^{\prime} \in t_{2}^{\prime}\left(i_{2}^{\prime}, i_{2}^{\prime \prime}\right): m^{\prime \prime}=\left(m_{1} \star m_{1}^{\prime}\right) \&\left(m_{2} \star m_{2}^{\prime}\right)\right\} \\
= & \left(\left(t_{1} \star t_{1}^{\prime}\right) \&\left(t_{2} \star t_{2}^{\prime}\right)\right)\left(\left(i_{1}, i_{2}\right),\left(i_{1}^{\prime \prime}, i_{2}^{\prime \prime}\right)\right)
\end{aligned}
$$

where we have used fact $4.30,(2)$.

Also we have that

$$
\mathrm{P}\left(t_{1} \star t_{2}\right)=\mathrm{P}\left(t_{1}\right) \star \mathrm{P}\left(t_{2}\right)
$$

This holds only because we demand transitions to be non-increasing. 


\subsection{The level 0 rules}

For each $G \in U$, there exists a rule $t(G) \in \mathcal{R} \mathcal{U}_{0}$ from $C a_{G}$ to $c(G)$, where $c(G)=\{c(G, i) \mid i \in O I(G)\}$. Here $m \in t(G)(i)$ iff $m \in t(G, i)$.

\subsection{Unfolding at level 1}

We now define what it means for a transition $t$ from $C$ to $C^{\prime}$ to be a level $1 c$ unfolding step ${ }^{10}$, to be written $1 c \vdash_{u}^{S} t: C \rightarrow C^{\prime}$.

$$
\begin{aligned}
& \left.\frac{t(G) \in \mathcal{R} \mathcal{U}_{0}}{1 \vdash_{u}^{S} \mathcal{I}_{s}(I d} C a_{H_{1}} \& t(G) \& I d C a_{H_{2}}\right) \\
& \frac{1 c \vdash_{u}^{S} I d_{C}}{\frac{1 c \vdash_{u}^{S} t_{1}, 1 c \vdash_{u}^{S} t_{2}}{1 c \vdash_{u}^{S} t_{1}+t_{2}}}
\end{aligned}
$$

Fact 7.12 If $1 c \vdash_{u}^{S} t$, then $1 c \vdash_{u}^{S} t \& I d_{-}$and $1 c \vdash_{u}^{S} I d_{-} \& t$. Also, $1 c \vdash_{u}^{S} \mathcal{I}_{s} t$.

Next we define what it means for a transition $t$ from $C$ to $C^{\prime}$ to be a level $1 c$ unfolding, to be written $1 c \vdash_{u} t: C \rightarrow C^{\prime}$ :

$$
\begin{aligned}
& \frac{1 c \vdash_{u}^{S} t: C \rightarrow C^{\prime}}{1 c \vdash_{u} \mathrm{P}(t): \mathrm{P}(C) \rightarrow \mathrm{P}\left(C^{\prime}\right)} \\
& \frac{1 c \vdash_{u} t: C \rightarrow C^{\prime}, 1 c \vdash_{u} t^{\prime}: C^{\prime} \rightarrow C^{\prime \prime}}{1 c \vdash_{u} t \star t^{\prime}: C \rightarrow C^{\prime \prime}}
\end{aligned}
$$

Observe that if $1 c \vdash_{u} t: C \rightarrow C^{\prime}$, then $t=\mathrm{P}(t), C=\mathrm{P}(C)$ and $C^{\prime}=\mathrm{P}\left(C^{\prime}\right)$.

Fact 7.13 If $1 c \vdash_{u} t_{1}$ and $1 c \vdash_{u} t_{2}$, then $1 c \vdash_{u} t_{1} \& t_{2}, 1 c \vdash_{u} t_{1}+t_{2}$ and $1 c \vdash_{u} \mathrm{P}\left(\mathcal{I}_{s}\left(t_{1}\right)\right)$.

PROOF: Inductions in the derivation tree:

- Concerning \&, it will be enough to show that $1 c \vdash_{u} t$ implies $1 c \vdash_{u}$ $t \& I d_{C}$ (and $1 c \vdash_{u} I d_{C} \& t$ ) for $C$ with $\mathrm{P}(C)=C$, as

$$
t_{1} \& t_{2}=\left(t_{1} \star I d_{-}\right) \&\left(I d_{-} \star t_{2}\right)=\left(t_{1} \& I d_{-}\right) \star\left(I d_{-} \& t_{2}\right)
$$

\footnotetext{
${ }^{10}$ The " $c$ " to denote that the complete search tree is modeled.
} 
If $t=t_{1} \star t_{2}$ with $1 c \vdash_{u} t_{1}, 1 c \vdash_{u} t_{2}$ this follows from

$t \& I d_{-}=\left(t_{1} \& I d_{-}\right) \star\left(t_{2} \& I d_{-}\right)$

If $t=\mathrm{P}\left(t^{\prime}\right)$ with $1 c \vdash_{u}^{S} t^{\prime}$, first note that by fact $7.121 c \vdash_{u}^{S} t^{\prime} \& I d_{-}$. Thus $1 c \vdash_{u} \mathrm{P}\left(t^{\prime} \& I d_{-}\right)$, i.e. $1 c \vdash_{u} t \& I d_{-}$.

- Concerning + , it will be enough to show that $1 c \vdash_{u} t^{\prime}$ implies $1 c \vdash_{u}$ $t+I d_{C}$ with $C$ such that $C=\mathrm{P}(C)-$ as

$t_{1}+t_{2}=\left(t_{1}+I d_{-}\right) \star\left(I d_{-}+t_{2}\right)$

If $t=t_{1} \star t_{2}$, this follows from

$t+I d_{-}=\left(t_{1}+I d_{-}\right) \star\left(t_{2}+I d_{-}\right)$

If $t=\mathrm{P}\left(t^{\prime}\right)$ with $1 c \vdash_{u}^{S} t^{\prime}$, first note that $1 c \vdash_{u}^{S} t^{\prime}+I d_{-}$.

Thus $1 c \vdash_{u} \mathrm{P}\left(t^{\prime}+I d_{-}\right)$, i.e. $1 c \vdash_{u} t+I d_{-}$.

- Concerning $\mathcal{I}_{-}\left(\right.$- $_{-}$, first suppose $1 c \vdash_{u} t$ because $t=\mathrm{P}\left(t^{\prime}\right), 1 c \vdash_{u}^{S} t^{\prime}$. Now also $1 c \vdash_{u}^{S} \mathcal{I}_{s}\left(t^{\prime}\right)$, and thus $1 c \vdash_{u} \mathrm{P}\left(\mathcal{I}_{s}\left(t^{\prime}\right)\right)$, i.e. also $1 c \vdash_{u}$ $\mathrm{P}\left(\mathcal{I}_{s}(t)\right)$.

Next suppose $t=t_{1} \star t_{2}$. By induction, $1 c \vdash_{u} \mathrm{P}\left(\mathcal{I}_{s}\left(t_{1}\right)\right)$ and $1 c \vdash_{u}$ $\mathrm{P}\left(\mathcal{I}_{s}\left(t_{2}\right)\right)$. The claim now follows from

$$
\mathrm{P}\left(\mathcal{I}_{s}\left(t_{1}\right)\right) \star \mathrm{P}\left(\mathcal{I}_{s}\left(t_{2}\right)\right)=\mathrm{P}\left(\mathcal{I}_{s}\left(t_{1} \star t_{2}\right)\right)
$$

If $1 c \vdash_{u} t$, there exists $t_{1} \ldots t_{n}$ such that $t=\mathrm{P}\left(t_{1}\right) \star \ldots \star \mathrm{P}\left(t_{n}\right)$ with $1 c \vdash_{u}^{S} t_{i}$ for $i=1 \ldots n$. Again, we can define the length of a transition $t$ as the minimal $n$ which can be used $(n \geq 1)$.

Observation 7.14 Suppose $1 c \vdash_{u} t: B \rightarrow C$, with $C=(I, B)$. Then for all $i \in I$ there exists $t_{i}$ such that $1 \vdash_{u} t_{i}: B \rightarrow B(i)$. We say that $t_{i}=\pi_{i}(t)$. 


\section{The diamond lemma, revisited}

Lemma 7.15 Suppose $1 c \vdash_{u}^{S} t_{1}: C \rightarrow C_{1}$ and $1 c \vdash_{u}^{S} t_{2}: C \rightarrow C_{2}$. Then there exists $C^{\prime}$, transition $t_{1}^{\prime}$ with $1 c \vdash_{u}^{S} t_{1}^{\prime}: C_{1} \rightarrow C^{\prime}$ and transition $t_{2}^{\prime}$ with $1 c \vdash_{u}^{S} t_{2}^{\prime}: C_{2} \rightarrow C^{\prime}$ such that $t_{1} \star t_{1}^{\prime}=t_{2} \star t_{2}^{\prime}$.

Proof: Much as the proof of lemma 5.2. A brief sketch: we can assume $C$ to consist of a single basic configuration (if $C$ is the union of several basic configurations each of these can be "treated" separately).

If $t_{1}=I d_{C}$, choose $t_{1}^{\prime}=t_{2}, t_{2}^{\prime}=I d_{C_{2}}$. Similarly if $t_{2}=I d_{C}$. So in the following we can assume that $t_{1}$ as well as $t_{2}$ are derived by means of (21). If "the same $G$ " is unfolded, we take $t_{1}^{\prime}=t_{2}^{\prime}=I d_{C_{1}}$. Otherwise, we can - dispensing with the $I d d_{C a_{H}}$-parts - write $t_{1}=\mathcal{I}_{s}\left(I d_{C a_{G_{1}}} \& t\left(G_{2}\right)\right)$, $t_{2}=\mathcal{I}_{s}\left(t\left(G_{1}\right) \& I d C_{G_{2}}\right)$. Then $C_{1}$ and $C_{2}$ take the form

$C_{1}=\left\{\mathcal{I}_{s}\left(C a_{G_{1}} \& c\left(G_{2}, i\right)\right) \mid i \in O I\left(G_{2}\right)\right\}$

$C_{2}=\left\{\mathcal{I}_{s}\left(c\left(G_{1}, i\right) \& C a_{G_{1}}\right) \mid i \in O I\left(G_{1}\right)\right\}$

Now define $t_{1}^{\prime}, t_{2}^{\prime}$ as follows:

$t_{1}^{\prime}=\mathcal{I}_{s}\left(t\left(G_{1}\right) \& I d_{c\left(G_{2}\right)}\right.$

$\left.t_{2}^{\prime}=\mathcal{I}_{s}\left(I_{c\left(G_{1}\right)} \& t\left(G_{2}\right)\right)\right\}$

That e.g. $1 c \vdash_{u}^{S} t_{1}^{\prime}$ follows from the fact that $t_{1}^{\prime}$ can be written on the form

$$
t_{1}^{\prime}=\sum_{i \in O I\left(G_{2}\right)} \mathcal{I}_{s}\left(t\left(G_{1}\right) \& I d_{c\left(G_{2}, i\right)}\right.
$$

where again $c\left(G_{2}, i\right)$ can be written on the form $\mathcal{I}_{s^{\prime}}\left(C a_{H^{\prime}}\right)$.

That $t_{1} \star t_{1}^{\prime}=t_{2} \star t_{2}^{\prime}$ follows from the fact that both equal $\mathcal{I}_{s}\left(t\left(G_{1}\right) \& t\left(G_{2}\right)\right)$.

\section{The Church-Rosser lemma, revisited}

Lemma 7.16 Suppose $1 c \vdash_{u} t_{1}: C \rightarrow C_{1}$ and $1 c \vdash_{u} t_{12}: C \rightarrow C_{2}$. Then there exists $C_{3}$ and transitions $t_{3}, t_{4}$ such that $1 c \vdash_{u} t_{3}: C_{1} \rightarrow C_{3}$, $1 c \vdash_{u} t_{4}: C_{2} \rightarrow C_{3}$, and such that $t_{1} \star t_{3}=t_{2} \star t_{4}$. 
Moreover, the length of $t_{3}$ is less than or equal the length of $t_{2}$, and the length of $t_{4}$ is less than or equal the length of $t_{1}$.

Proof: We use induction in the length of $t_{1}$ plus the length of $t_{2}$. For the induction step, proceed as in the proof of lemma 5.3. So assume that both transitions have length 1 . The situation is as follows: we have $1 c \vdash_{u}^{S} t_{1}^{\prime}: C^{\prime} \rightarrow C_{1}^{\prime}$ and $1 c \vdash_{u}^{S} t_{2}^{\prime}: C^{\prime \prime} \rightarrow C_{2}^{\prime}$, with $t_{1}=\mathrm{P}\left(t_{1}^{\prime}\right), t_{2}=\mathrm{P}\left(t_{2}^{\prime}\right)$, and $C=\mathrm{P}\left(C^{\prime}\right)=\mathrm{P}\left(C^{\prime \prime}\right)$. We can assume that $C^{\prime}=C^{\prime \prime}$, as we can "expand" $t_{1}^{\prime}$ and $t_{2}^{\prime}$. Now apply lemma 7.15, to find $t_{3}^{\prime}, t_{4}^{\prime}$ and $C_{3}^{\prime}$ with $1 c \vdash_{u}^{S} t_{3}^{\prime}: C_{1}^{\prime} \rightarrow C_{3}^{\prime}, 1 c \vdash_{u}^{S} t_{4}^{\prime}: C_{2}^{\prime} \rightarrow$ $C_{3}^{\prime}, t_{1}^{\prime} \star t_{3}^{\prime}=t_{2}^{\prime} \star t_{4}^{\prime}$. Then define $C_{3}=\mathrm{P}\left(C_{3}^{\prime}\right), t_{3}=\mathrm{P}\left(t_{3}^{\prime}\right), t_{4}=\mathrm{P}\left(t_{4}^{\prime}\right)$. Then $1 c \vdash_{u} t_{3}, 1 c \vdash_{u} t_{4}$, and

$t_{1} \star t_{3}=\mathrm{P}\left(t_{1}^{\prime}\right) \star \mathrm{P}\left(t_{3}^{\prime}\right)=\mathrm{P}\left(t_{1}^{\prime} \star t_{3}^{\prime}\right)=\mathrm{P}\left(t_{2}^{\prime} \star t_{4}^{\prime}\right)=t_{2} \star t_{4}$

\subsection{Level 1 semantics}

We say that a configuration $C=(I, B)$ is in normal form iff $C$ is pruned and for all $i \in I B(i)$ is empty.

Lemma 7.16, together with the observation that if $C$ is in normal form and $1 c \vdash_{u} t: C \rightarrow C^{\prime}$ then $C=C^{\prime}$, shows that the following is welldefined:

Definition 7.17 Given basic configuration $B$ (not failure). Suppose $1 c \vdash_{u}$ $t: B \rightarrow C$ with $C$ in normal form. Then $\llbracket B \rrbracket_{1}=C$.

If no such $t$ and $C$ exists, $\llbracket B \rrbracket_{1}=\perp$.

Fact $7.18 \llbracket B \rrbracket_{1}=\perp$ iff $B$ loops at level 1 by a fair strategy (as defined in definition 5.7)

Proof: Suppose $\llbracket B \rrbracket_{1}=\perp$. Given $n \geq 0$. It is easily seen that there will exist $t_{n}$ and $C^{\prime}=\left(I^{\prime}, B^{\prime}\right)$ such that $1 c \vdash_{u} t_{n}: B \rightarrow C^{\prime}$, and such that for all $i \in I^{\prime}$ we have $1 \vdash_{u} \pi_{i}\left(t_{n}\right): B \rightarrow B^{\prime}(i)$ where either $B^{\prime}(i)$ is empty or $\pi_{i}\left(t_{n}\right)$ is composed of $n$ fair level 1 unfolding steps. Now, there exists at least one $i \in I^{\prime}$ where $B^{\prime}(i)$ is not empty (otherwise $C^{\prime}$ would be in normal form). Hence we conclude that $B$ loops at level 1 by a fair strategy.

Conversely, suppose there exists $C$ in normal form such that $1 c \vdash_{u} t: B \rightarrow$ $C$. Then there exists U-forests $f_{1} \ldots f_{k}$ such that $\mathcal{U}_{f_{i}}(B)$ is either empty or failure for all $i$. Moreover, there exists a $n$ such that for all U-forests $f$ where 
the shortest working path is longer than $n$, there exists an $i$ such that $f$ can be written as $f_{i} \star f^{\prime}$ for some $f^{\prime}$. Hence for such $f$ also $\mathcal{U}_{f}(B)$ is empty or failure, showing that $B$ does not loop at level 1 by a fair strategy.

\section{$\mathcal{L} \mathcal{R}$ semantics}

We say that $t$ is a $\mathcal{L} \mathcal{R}$ level $1 c$ single step if $t$ takes the form $t=\mathcal{I}_{s}\left(t(G) \& I d_{B}\right)$, with $t(G) \in \mathcal{R} \mathcal{U}_{0}$. We say that $t$ is a $\mathcal{L} \mathcal{R}$ level $1 c$ step if $t$ takes the form $t=t_{1}+\cdots+t_{k}$, at least one $t_{i}$ being a $\mathcal{L} \mathcal{R}$ level $1 c$ single step and the rest being of form $I d_{B}, B$ empty. We say that $t$ is a $\mathcal{L R}$ level $1 c$ unfolding if $t$ takes the form $t=\mathrm{P}\left(t_{1}\right) \star \cdots \star \mathrm{P}\left(t_{i}\right)$, each $t_{i}$ being a $\mathcal{L} \mathcal{R}$ level $1 c$ step.

Definition 7.19 Given basic configuration $B$. Suppose $1 c \vdash_{u} t: B \rightarrow C$ with $C$ in normal form, where $t$ is a $\mathcal{L} \mathcal{R}$ level $1 c$ unfolding. Then $\llbracket B \rrbracket_{1}^{L}=C$.

If no such $t$ and $C$ exists, $\llbracket B \rrbracket_{1}=\perp$.

Fact $7.20 \llbracket B \rrbracket_{1}^{L}=\perp$ iff $B$ loops at level 1 by a $\mathcal{L} \mathcal{R}$ strategy (as defined in definition 5.10).

Proof: Suppose $\llbracket B \rrbracket_{1}^{L}=\perp$. Given $n \geq 0$. It is easily seen that there will exist $t_{n}$ and $C^{\prime}=\left(I^{\prime}, B^{\prime}\right)$ such that $1 c \vdash_{u} t_{n}: B \rightarrow C^{\prime}$, and such that for all $i \in I^{\prime}$ we have $1 \vdash_{u} \pi_{i}\left(t_{n}\right): B \rightarrow B^{\prime}(i)$ where either $B_{i}^{\prime}$ is empty or $\pi_{i}\left(t_{n}\right)$ is composed of $n \mathcal{L} \mathcal{R}$ level 1 unfolding steps. Now, there exists at least one $i \in I^{\prime}$ where $B^{\prime}(i)$ is not empty (otherwise $C^{\prime}$ would be in normal form). Hence we conclude that $B$ loops at level 1 by a $\mathcal{L} \mathcal{R}$ strategy.

Conversely, suppose there exists $C$ in normal form such that $1 c \vdash_{u} t$ : $B \rightarrow C$, with $t$ a $\mathcal{L} \mathcal{R}$ level $1 c$ unfolding. Then there exists U-forests $f_{1} \ldots f_{k}$ such that $\mathcal{U}_{f_{i}}(B)$ is either empty or failure for all $i$. Moreover, there exists a $n$ such that for all $\mathcal{L} \mathcal{R}$ U-forests $f$ of size greater than $n$, there exists an $i$ such that $f$ can be written as $f_{i} \star f^{\prime}$ for some $f^{\prime}$. Hence for such $f$ also $\mathcal{U}_{f}(B)$ is empty or failure, showing that $B$ does not loop at level 1 by a $\mathcal{L} \mathcal{R}$ strategy.

\subsection{Folding at level 1}

We now define what it means for a transition $t$ from $C$ to $C^{\prime}$ to be a level $1 c$ transition, to be written $1 \vdash_{f}^{S} t: C \rightarrow C^{\prime}$. 
We will assume the existence of a partial (perhaps multivalued) function $s(G)$ such that $\mathrm{P}\left(\mathcal{I}_{s(G)}(t(G))\right)$ is reversible.

$$
\begin{aligned}
& \left.\frac{t(G) \in \mathcal{R} \mathcal{U}_{0}}{1 c \vdash_{f}^{S} \mathcal{I}_{s}\left(I d a _ { H _ { 1 } } \& \mathcal { R } \left(\mathrm{P}\left(\mathcal{I}_{s(G)}(t(G))\right) \& I d\right.\right.} C a_{H_{2}}\right) \\
& \frac{1 c \vdash_{f}^{S} I d_{C}}{1 c \vdash_{f}^{S} t_{1}, 1 c \vdash_{f}^{S} t_{2}} \\
& 1 c \vdash_{f}^{S} t_{1}+t_{2}
\end{aligned}
$$

Next we define what it means for a transition $t$ from $C$ to $C^{\prime}$ to be a level $1 c$ folding, to be written $1 c \vdash_{f} t: C \rightarrow C^{\prime}$ :

$$
\begin{aligned}
& \frac{1 c \vdash_{f}^{S} t: C \rightarrow C^{\prime}}{1 c \vdash_{f} \mathrm{P}(t): \mathrm{P}(C) \rightarrow \mathrm{P}\left(C^{\prime}\right)} \\
& \frac{1 c \vdash_{f} t: C \rightarrow C^{\prime}, 1 c \vdash_{f} t^{\prime}: C^{\prime} \rightarrow C^{\prime \prime}}{1 c \vdash_{f} t \star t^{\prime}: C \rightarrow C^{\prime \prime}}
\end{aligned}
$$

\subsection{Unfold/fold at level 1}

Next we now define what it means for a transition $t$ from $C$ to $C^{\prime}$ to be a level $1 c$ folding, to be written $1 c \vdash t: B \rightarrow B^{\prime}$.

$$
\begin{aligned}
& \frac{1 c \vdash_{u}^{S} t: C \rightarrow C^{\prime}}{1 c \vdash \mathrm{P}(t): \mathrm{P}(C) \rightarrow \mathrm{P}\left(C^{\prime}\right)} \\
& \frac{1 c \vdash_{f}^{S} t: C \rightarrow C^{\prime}}{1 c \vdash \mathrm{P}(t): \mathrm{P}(C) \rightarrow \mathrm{P}\left(C^{\prime}\right)} \\
& \frac{1 c \vdash t: C \rightarrow C^{\prime}, 1 c \vdash t^{\prime}: C^{\prime} \rightarrow C^{\prime \prime}}{1 c \vdash t \star t^{\prime}: C \rightarrow C^{\prime}}
\end{aligned}
$$

Fact 7.21 If $1 c \vdash t_{1}$ and $1 c \vdash t_{2}$, then also $1 c \vdash t_{1} \& t_{2}$ and $1 c \vdash \mathrm{P}\left(\mathcal{I}_{s}\left(t_{1}\right)\right)$.

\section{The switching lemma, revisited}

Lemma 7.22 Suppose $1 c \vdash t_{1}: C_{1} \rightarrow C$ is a folding step, i.e. is derived by means of rule (32), and suppose $1 c \vdash t_{2}: C \rightarrow C_{2}$ is an unfolding step, i.e. is derived by means of rule (31). Then there exists $t_{3}, t_{4}$ and $C_{3}$ such that 
$1 c \vdash t_{31}: C_{1} \rightarrow C_{3}$ by an unfolding step; $1 c \vdash t_{4}: C_{3} \rightarrow C_{2}$ by a folding step; and $t_{1} \star t_{2}=t_{3} \star t_{4}$.

Proof: (A sketch only) There exists $t_{1}^{\prime}, t_{2}^{\prime}, C_{1}^{\prime}, C_{2}^{\prime}, C^{\prime}$ and $C^{\prime \prime}$ such that $1 c \vdash_{f}^{S} t_{1}^{\prime}: C_{1}^{\prime} \rightarrow C^{\prime} .1 c \vdash_{u}^{S} t_{2}^{\prime}: C^{\prime \prime} \rightarrow C_{2}^{\prime}, t_{1}=\mathrm{P}\left(t_{1}^{\prime}\right), t_{2}=\mathrm{P}\left(t_{2}^{\prime}\right)$ and $C=\mathrm{P}\left(C^{\prime}\right)=\mathrm{P}\left(C^{\prime \prime}\right)$. It is not hard to see that we can assume that $t_{1}^{\prime}$ and $t_{2}^{\prime}$ are derived by means of (26) and (21) respectively, and that we can assume $C$ to be a singleton (i.e. not $\emptyset$ ).

There are two cases (where we dispense with writing the $I d C_{H}$-parts):

1. $t_{1}^{\prime}, t_{2}^{\prime}$ takes the form

$$
\begin{aligned}
t_{1}^{\prime} & =\mathcal{I}_{s_{1}}\left(\mathcal{R}\left(\mathrm{P}\left(\mathcal{I}_{s(G)}(t(G))\right)\right)\right) \\
t_{2}^{\prime} & =\mathcal{I}_{s_{2}}(t(G))
\end{aligned}
$$

Thus $t_{1}=\mathcal{R}\left(\mathrm{P}\left(\mathcal{I}_{s_{1} \star s(G)}(t(G))\right)\right), t_{2}=\mathrm{P}\left(\mathcal{I}_{s_{2}}(t(G))\right)$. Then

$\mathcal{I}_{s_{1} \star s(G)}\left(C a_{G}\right)=C=\mathcal{I}_{s_{2}}\left(C a_{G}\right)$

enabling us to conclude that $s_{1} \star s(G)=s_{2}$ and hence also $C_{1}=C_{2}$. Now we can use $t_{3}=t_{4}=I d_{C_{1}}$. That $t_{1} \star t_{2}=I d_{C_{1}}$ is an easy consequence of fact $4.30,(4)$.

2. $t_{1}^{\prime}, t_{2}^{\prime}$ takes the form

$$
\begin{aligned}
t_{1}^{\prime} & =\mathcal{I}_{s_{1}}\left(\mathcal{R}\left(\mathrm{P}\left(\mathcal{I}_{s\left(G_{1}\right)}\left(t\left(G_{1}\right)\right)\right)\right) \& I d_{C a_{G_{2}}}\right) \\
t_{2}^{\prime} & =\mathcal{I}_{s_{2}}\left(I d_{C a_{G_{1}}} \& t\left(G_{2}\right)\right)
\end{aligned}
$$

Now apply the usual technique: we infer that $s_{1} \star\left(s\left(G_{1}\right) \& I d_{-}\right)=s_{2}$, and define

$$
\begin{aligned}
t_{4}^{\prime} & =\mathcal{I}_{s_{1}}\left(\mathcal{R}\left(\mathrm{P}\left(\mathcal{I}_{s\left(G_{1}\right)}\left(t\left(G_{1}\right)\right)\right)\right) \& I d_{c\left(G_{2}\right)}\right) \\
t_{3}^{\prime} & =\mathcal{I}_{s_{2}}\left(I_{c\left(G_{1}\right)} \& t\left(G_{2}\right)\right)
\end{aligned}
$$

We now define $t_{3}=\mathrm{P}\left(t_{3}^{\prime}\right), t_{4}=\mathrm{P}\left(t_{4}^{\prime}\right)$. Clearly $1 c \vdash_{u}^{S} t_{3}^{\prime}, 1 c \vdash_{f}^{S} t_{4}^{\prime}$ and

$$
t_{3} \star t_{4}=\mathrm{P}\left(t_{3}^{\prime} \star t_{4}^{\prime}\right)=\mathrm{P}\left(t_{1}^{\prime} \star t_{2}^{\prime}\right)=t_{1} \star t_{2}
$$




\section{The normalization lemma, revisited}

Lemma 7.23 Suppose $1 c \vdash t: C^{\prime} \rightarrow C^{\prime}$. Then there exists $t_{1}, t_{2}, C^{\prime \prime}$ such that $1 c \vdash_{u} t_{1}: C \rightarrow C^{\prime \prime}, 1 c \vdash_{f} t_{2}: C^{\prime \prime} \rightarrow C^{\prime}, t=t_{1} \star t_{2}$.

Proof: As the proof of lemma 5.15, now exploiting lemma 7.22.

\subsection{Unfolding at level 2}

Now assume that we have defined $\mathcal{R} \mathcal{U}_{1}$, a finite set of rules at level 1 . Assume that there is a bijective correspondence between $\mathcal{R} \mathcal{U}_{1}$ and $U$, such that the rule corresponding to $G$ is a transition from $C a_{G}$. Then there is no risk of a configuration being stuck (i.e. not in normal form but cannot be unfolded further).

We now define what it means for a transition $t$ from $C$ to $C^{\prime}$ to be a level $2 c$ unfolding step, to be written $2 c \vdash^{S} t: C \rightarrow C^{\prime}$.

$$
\begin{aligned}
& \left.\frac{t \in \mathcal{R U}_{1}}{2 c \vdash^{S} \mathcal{I}_{s}\left(i d a_{H_{1}} \& t \& i d\right.} C a_{H_{2}}\right) \\
& \frac{2 c \vdash^{S} I d_{C}}{\frac{2 c \vdash^{S} t_{1}, 2 c \vdash^{S} t_{2}}{2 c \vdash^{S} t_{1}+t_{2}}}
\end{aligned}
$$

Next we define what it means for a transition $t$ from $C$ to $C^{\prime}$ to be a level $2 c$ unfolding, to be written $2 c \vdash t: C \rightarrow C^{\prime}$ :

$$
\begin{aligned}
& \frac{2 c \vdash^{S} t: C \rightarrow C^{\prime}}{2 c \vdash \mathrm{P}(t): \mathrm{P}(C) \rightarrow \mathrm{P}\left(C^{\prime}\right)} \\
& \frac{2 c \vdash t: C \rightarrow C^{\prime}, 21 c \vdash t^{\prime}: C^{\prime} \rightarrow C^{\prime \prime}}{2 c \vdash t \star t^{\prime}: C \rightarrow C^{\prime}}
\end{aligned}
$$

Fact 7.24 If $2 c \vdash t: C \rightarrow C^{\prime}$, also $1 c \vdash t: C \rightarrow C^{\prime}$.

$$
\text { If } 2 c \vdash^{S} t: C \rightarrow C^{\prime} \text {, also } 1 c \vdash \mathrm{P}(t): \mathrm{P}(C) \rightarrow \mathrm{P}\left(C^{\prime}\right) \text {. }
$$

PRoOF: Induction in the derivation tree: the only interesting case is where (34) has been applied. We must show that 
$1 c \vdash \mathrm{P}\left(\mathcal{I}_{s}\left(I_{C a_{H_{1}}} \& t \& I d C_{H_{2}}\right)\right)$

But this is a consequence of fact 7.21 .

By combining lemma 7.23 and fact 7.24 we get

Fact 7.25 If $2 c \vdash t: C \rightarrow C^{\prime}$, there exists $t_{1}, t_{2}, C^{\prime \prime}$ such that

$1 c \vdash_{u} t_{1}: C \rightarrow C^{\prime \prime}, 1 c \vdash_{f} t_{2}: C^{\prime \prime} \rightarrow C^{\prime}, t=t_{1} \star t_{2}$.

If $C^{\prime}$ is in normal form, $C^{\prime}=C^{\prime \prime}$.

\subsection{Level 2 semantics}

Definition 7.26 Given basic configuration $B$ (not failure). Suppose $2 c \vdash$ $t: B \rightarrow C$ with $C$ in normal form. Then $\llbracket B \rrbracket_{2}=C$.

If no such $t$ and $C$ exists, $\llbracket B \rrbracket_{2}=\perp$.

By fact 7.25, this is well-defined.

We say that $t$ is a fair level $2 c$ single step if $t$ takes the form $t=\mathcal{I}_{s}\left(t_{1} \& \cdots\right.$ $\left.\& t_{n}\right), n \geq 1$, each $t_{i} \in \mathcal{R} \mathcal{U}_{1}$. We say that $t$ is a fair level $2 c$ step if $t$ takes the form $t=t_{1}+\cdots+t_{k}$, at least one $t_{i}$ being a fair level $2 c$ single step and the rest of form $I d_{B}$ with $B$ empty. We say that $t$ is a fair level $2 c$ unfolding if $t$ takes the form $t=\mathrm{P}\left(t_{1}\right) \star \cdots \star \mathrm{P}\left(t_{k}\right)$, each $t_{i}$ being a fair level $2 c$ step. We say that $B$ loops at level $2 c$ by a fair strategy if for all $i>0$ there exists $C_{i}$ not in normal form and fair level $2 c$ step $t_{i}$ from $C_{i-1}$ to $C_{i}$ (here $C_{0}=B$ ).

We say that $t$ is a $\mathcal{L R}$ level $2 c$ single step if $t$ takes the form $t=\mathcal{I}_{s}\left(t_{1} \& I d_{B}\right)$, with $t_{1} \in \mathcal{R} \mathcal{U}_{1}$. We say that $t$ is a $\mathcal{L R}$ level $2 c$ step if $t$ takes the form $t=t_{1}+\cdots+t_{k}$, at least one $t_{i}$ being a $\mathcal{L R}$ level 2 single step and the rest of form $I d_{B}$ with $B$ empty. We say that $t$ is a $\mathcal{L R}$ level $2 c$ unfolding if $t$ takes the form $t=\mathrm{P}\left(t_{1}\right) \star \cdots \star \mathrm{P}\left(t_{k}\right)$, each $t_{i}$ being a $\mathcal{L R}$ level $2 c$ step. We say that $B$ loops at level $2 c$ by the $\mathcal{L R}$ strategy if for all $i>0$ there exists $C_{i}$ not in normal form and $\mathcal{L R}$ level $2 c$ step $t_{i}$ from $C_{i-1}$ to $C_{i}$ (here $C_{0}=B$ ).

Definition 7.27 Given basic configuration $B$ (not failure) Suppose $2 c \vdash$ $t: B \rightarrow C$ with $C$ in normal form, where $t$ is a $\mathcal{L R}$ level $2 c$ unfolding. Then $\llbracket B \rrbracket_{2}^{L}=C$. 
If no such $t$ and $C$ exists, $\llbracket B \rrbracket_{2}^{L}=\perp$.

Clearly, $\llbracket B \rrbracket_{2}^{L}=\perp$ iff $B$ loops at level $2 c$ by the $\mathcal{L R}$ strategy.

\subsection{Total correctness}

Theorem 7.28 Assume all U-mirrors occurring in rules in $\mathcal{R} \mathcal{U}_{1}$ satisfy $\mathcal{F}(1)$. Then for all $B, \llbracket B \rrbracket_{2}=\llbracket B \rrbracket_{1}$.

Proof: First suppose $\llbracket B \rrbracket_{2}=C \neq \perp$. By fact 7.25 , also $\llbracket B \rrbracket_{1}=C$.

Now suppose $\llbracket B \rrbracket_{2}=\perp$. Then for all $n \geq 1$ there will exist fair level $2 c$ step $t_{n}$ and $C_{n}$ not in normal form such that $2 c \vdash \mathrm{P}\left(t_{n}\right): C_{n-1} \rightarrow C_{n}$ $\left(C_{0}=B\right)$. Let $t_{n}^{\prime}=\mathrm{P}\left(t_{1}\right) \star \cdots \star \mathrm{P}\left(t_{k}\right) .2 c \vdash t_{n}^{\prime}: B \rightarrow C_{n}$, and by fact 7.25 there exists $t_{n}^{\prime \prime}, t_{n}^{\prime \prime \prime}$ and $C_{n}^{\prime}$ such that $1 c \vdash_{u} t_{n}^{\prime \prime}: B \rightarrow C_{n}^{\prime}, 1 c \vdash_{f} t_{n}^{\prime \prime \prime}: C_{n}^{\prime} \rightarrow C_{n}$ and $t_{n}^{\prime}=t_{n}^{\prime \prime} \star t_{n}^{\prime \prime \prime}$.

As $C_{n}$ contains a non-empty basic configuration, this shows that $t_{n}^{\prime}$ for all $n$ contains at least one mirror from $B$ to a non-empty basic configuration. Then it will be possible (by Königs lemma) for all $n$ to find $m_{n} \in t_{n}$ such that $m_{n}^{\prime}=m_{1} \star \cdots \star m_{n}$ is a mirror in $t_{n}^{\prime}$ from $B$ to a non-empty basic configuration. Also there will exist mirrors $m_{n}^{\prime \prime} \in t_{n}^{\prime \prime}$ and $m_{n}^{\prime \prime \prime} \in t_{n}^{\prime \prime \prime}$ such that $m_{n}^{\prime}=m_{n}^{\prime \prime} \star m_{n}^{\prime \prime \prime}$. It is easily seen that $1 \vdash_{u} m_{n}^{\prime \prime}: B \rightarrow B_{n}^{\prime}$, with $B_{n}^{\prime}$ not failure.

Due to the assumption of the theorem, each $m_{i}$ will satisfy $\mathcal{F}(1)$. Then, by lemma 4.32, each $m_{n}^{\prime}$ will satisfy $\mathcal{F}(n)$. But then also $m_{n}^{\prime \prime}$ will satisfy $\mathcal{F}(n)$. By lemma 5.8 , this shows that $B$ loops at level 1 by a fair strategy, and by fact $7.18 \llbracket B \rrbracket_{1}=\perp$.

Theorem 7.29 Assume all U-mirrors occurring in rules in $\mathcal{R} \mathcal{U}_{1}$ satisfy $\mathcal{L}(1)$. Then for all $B, \llbracket B \rrbracket_{2}^{L} \geq \llbracket B \rrbracket_{1}^{L}$.

Proof: First suppose $\llbracket B \rrbracket_{2}^{L}=C \neq \perp$. Then also $\llbracket B \rrbracket_{2}=C$, so by fact $7.25 \llbracket B \rrbracket_{1}=C$. Now either $\llbracket B \rrbracket_{1}^{L}=C$ or $\llbracket B \rrbracket_{1}^{L}=\perp$.

Now suppose $\llbracket B \rrbracket_{2}^{L}=\perp$. Then for all $n \geq 1$ there will exist $\mathcal{L R}$ level $2 c$ step $t_{n}$ and $C_{n}$ not in normal form such that $2 c \vdash \mathrm{P}\left(t_{n}\right): C_{n-1} \rightarrow C_{n}$ $\left(C_{0}=B\right)$. Let $t_{n}^{\prime}=\mathrm{P}\left(t_{1}\right) \star \cdots \star \mathrm{P}\left(t_{n}\right) .2 c \vdash t_{n}^{\prime}: B \rightarrow C_{n}$, and by fact 7.25 there exists $t_{n}^{\prime \prime}, t_{n}^{\prime \prime \prime}$ and $C_{n}^{\prime}$ such that $1 c \vdash_{u} t_{n}^{\prime \prime}: B \rightarrow C_{n}^{\prime}, 1 c \vdash_{f} t_{n}^{\prime \prime \prime \prime}: C_{n}^{\prime} \rightarrow C_{n}$ and $t_{n}^{\prime}=t_{n}^{\prime \prime} \star t_{n}^{\prime \prime \prime}$.

As $C_{n}$ contains a non-empty basic configuration, this shows that $t_{n}^{\prime}$ for all $n$ contains at least one mirror from $B$ to a non-empty basic configuration. 
Then it will be possible for all $n$ to find $m_{n} \in t_{n}$ such that $m_{n}^{\prime}=m_{1} \star \cdots m_{n}$ is a mirror in $t_{n}^{\prime}$ from $B$ to a non-empty basic configuration. Also there will exist mirrors $m_{n}^{\prime \prime} \in t_{n}^{\prime \prime}$ and $m_{n}^{\prime \prime \prime} \in t_{n}^{\prime \prime \prime}$ such that $m_{n}^{\prime}=m_{n}^{\prime \prime} \star m_{n}^{\prime \prime}$. It is easily seen that $1 \vdash_{u} m_{n}^{\prime \prime}: B \rightarrow B_{n}^{\prime}$, with $B_{n}^{\prime}$ not failure.

Due to the assumption of the theorem, each $m_{i}$ satisfies $\mathcal{L}(1)$. By lemma 4.37, there exists an increasing sequence

$m_{1}^{\prime} \prec m_{2}^{\prime} \prec m_{3}^{\prime} \prec \cdots$

Now two possibilities:

1. $E\left(m_{n}^{\prime}\right)$ is not bounded. Then neither $E\left(m_{n}^{\prime \prime}\right)$ is bounded, so by lemma $5.11 B$ loops at level 1 by a $\mathcal{L} \mathcal{R}$ strategy.

2. $E\left(m_{n}^{\prime}\right)$ is bounded. Then $L\left(m_{n}^{\prime}\right)$ is unbounded, so also $L\left(m_{n}^{\prime \prime}\right)$ is unbounded. Then again lemma 5.11 tells us that $B$ loops at level 1 by a $\mathcal{L} \mathcal{R}$ strategy.

In both cases, fact 7.20 tells us that $\llbracket B \rrbracket_{1}^{L}=\perp$.

\section{References}

[Amt92] Torben Amtoft. Unfold/fold transformations preserving termination properties. To appear in the proceedings of PLILP 92, 1992.

[BD77] R.M. Burstall and John Darlington. A transformation system for developing recursive programs. Journal of the ACM, 24(1):44-67, January 1977.

[DP88] John Darlington and Helen Pull. A program development methodology based on a unified approach to execution and transformation. In D. Bjørner, A.P. Ershov, and N.D. Jones, editors, Partial Evaluation and Mixed Computation, pages 117-131. North-Holland, 1988.

[Gre87] Steve Gregory. Parallel Logic Programming in PARLOG - the language and its implementation. Addison-Wesley, 1987.

[GS91] P. A. Gardner and J. C. Shepherdson. Unfold/fold transformations of logic programs. In Computational Proofs: Essays in honour of Alan Robinson. 1991. 
[Han91] Torben Amtoft Hansen. Properties of unfolding-based meta-level systems. In Partial Evaluation and Semantics-Based Program manipulation, New Haven, Connecticut. (Sigplan Notices, vol. 26, no. 9), 1991.

[KK90] Tadashi Kawamura and Tadashi Kanamori. Preservation of stronger equivalence in unfold/fold logic program transformation. Theoretical Computer Science, 75:139-156, 1990.

[Kot85] Laurent Kott. Unfold/fold program transformations. In Maurice Nivat and John C. Reynolds, editors, Algebraic methods in Semantics, chapter 12. Cambridge University Press, 1985.

[Llo84] J. W. Lloyd. Foundations of Logic Programming. Springer-Verlag, 1984.

[NN90] Hanne Riis Nielson and Flemming Nielson. Eureka definitions for free! or disagreement points for fold/unfold transformations. In Neil D. Jones, editor, ESOP 90, Copenhagen, Denmark. LNCS 432, pages 291-305, May 1990.

[Pal89] Catuscia Palamidessi. Algebraic properties of idempotent substitutions. Technical Report TR-33/89, University of Piss, 1989.

[Plo81] Gordon D. Plotkin. A structural approach to operational semantics. Technical Report FN-19, DAIMI, University of Aarhus, Denmark, September 1981.

[PP91a] Maurizio Proietti and Alberto Pettorossi. Semantics preserving transformation rules for Prolog. In Partial Evaluation and SemanticsBased Program Manipulation, New Haven, Connecticut. (Sigplan Notices, vol. 26, no. 9), 1991.

[PP91b] Maurizio Proietti and Alberto Pettorossi. Unfolding - Definition Folding, in this order, for avoiding unnecessary variables in logic programs. In Proceedings of PLILP 91, Passau, Germany (LNCS 528), August 1991.

[Sek91] Hirohisa Seki. Unfold/fold transformations of stratified programs. Theoretical Computer Science, 86(1):107-139, 1991. 
[Søn89] Harald Søndergaard. Semantics-based analysis and transformation of logic programs. Technical Report 89/22, DIKU, University of Copenhagen, Denmark, 1989.

[TS84] Hisao Tamaki and Taisuke Sate. Unfold/fold transformation of logic programs. In Proceedings of 2nd International Logic Programming Conference, Uppsala, pages 127-138, 1984.

[Tur86] Valentin F. Turchin. The concept of a supercompiler. ACM Transactions on Programming Languages and Systems, 8(3):292-325, July 1986.

[Wad90] Philip Wadler. Deforestation: Transforming programs to eliminate trees. Theoretical Computer Science, 73:231-248, 1990. 